TRANSACTIONS OF THE

AMERICAN MATHEMATICAL SOCIETY

Volume 354, Number 3, Pages 1233-1264

S 0002-9947(01)02897-5

Article electronically published on October 26, 2001

\title{
SPHERICAL UNITARY HIGHEST WEIGHT REPRESENTATIONS
}

\author{
BERNHARD KRÖTZ AND KARL-HERMANN NEEB
}

\begin{abstract}
In this paper we give an almost complete classification of the $\mathrm{H}$ spherical unitary highest weight representations of a hermitian Lie group $G$, where $G / H$ is a symmetric space of Cayley type.
\end{abstract}

\section{INTRODUCTION}

Let $G / H$ be a semisimple symmetric space attached to an involution $\tau: G \rightarrow G$. Then in order for a unitary irreducible representation $(\pi, \mathcal{H})$ of $G$ to be realized in $L^{p}(G / H), 1 \leq p \leq \infty$, or more generally in $\mathcal{D}^{\prime}(G / H)$, it is necessary that $(\pi, \mathcal{H})$ is $H$-spherical, i.e., the space of $H$-invariant distribution vectors $\left(\mathcal{H}^{-\infty}\right)^{H}$ has to be non-zero.

Two classes of representations in the unitary dual $\widehat{G}$ of $G$ are of special interest: The unitary principal series and the discrete series. For the unitary principal series induced from a parabolic subgroup we have for almost all parameters a complete description of $\left(\mathcal{H}^{-\infty}\right)^{H}$ (cf. [1], 27] for the $\theta \tau$-stable minimal parabolics and [4] for the general case). Discrete series on $G / H$ were constructed in 12] (see also 30]) and in 3] it was shown that $\left(\mathcal{H}^{-\infty}\right)^{H}$ is one-dimensional for all discrete series on $G / H$ except for four types of exceptional symmetric spaces. Holomorphic discrete series, i.e., unitary highest weight representation of $G$ which can be realized in $L^{2}(G / H)$, were constructed in 28, 29] (this construction is essentially different from the one in [12]) and it was shown that $\left(\mathcal{H}^{-\infty}\right)^{H}$ is always one-dimensional (this also covers half of the exceptional spaces in [3]).

In this paper we address the problem of classifying all $H$-spherical unitary highest weight representations for a simply connected hermitian Lie group $G$. We write $\mathfrak{g}=\mathfrak{h} \oplus \mathfrak{q}$ for the $\tau$-eigenspace decomposition of $\mathfrak{g}:=\operatorname{Lie}(G)$ and choose a $\tau$-stable Cartan decomposition $\mathfrak{g}=\mathfrak{k} \oplus \mathfrak{p}$. Set $K:=\exp (\mathfrak{k})$. Note that $\mathfrak{z}(\mathfrak{k})$ is one-dimensional since $G$ is hermitian and that $H$ is connected since $G$ is simply connected. Now non-trivial $H$-spherical highest weight representations exist if and only if $\mathfrak{z}(\mathfrak{k}) \subseteq \mathfrak{q}$ which means that $G / H$ is compactly causal (cf. 18).

The unitary highest weight representations $\left(\pi_{\lambda}, \mathcal{H}_{\lambda}\right)$ of $G$ can be parametrized by the highest weight $\lambda$ of the corresponding finite-dimensional unitary representation $\left(\pi_{\lambda}^{K}, F(\lambda)\right)$ of $K$. In the scalar case, i.e., $\operatorname{dim} F(\lambda)=1$, the classification of unitary

Received by the editors March 7, 2001.

1991 Mathematics Subject Classification. Primary 22E46.

Key words and phrases. Highest weight representation, spherical representation.

Part of the work of the first author was supported by the Erwin-Schrödinger-Institut, Vienna, and NSF grant DMS-0097314.

Part of the work of the second author was done on a visit supported by the Research Institute of The Ohio State University. 
highest weight representations was accomplished in [33] and 32 while the general case has been treated in [9] and [20]. The generalized Verma module $N(\lambda)=$ $\mathcal{U}\left(\mathfrak{g}_{\mathbb{C}}\right) \otimes_{\mathcal{U}\left(\mathfrak{k}_{\mathbb{C}}+\mathfrak{p}^{+}\right)} F(\lambda)$ is a highest weight module for $\mathfrak{g}$ with highest weight $\lambda$. It has a unique irreducible quotient $L(\lambda)$ which is $(\mathfrak{g}, K)$-isomorphic to the space of $K$-finite vectors of $\left(\pi_{\lambda}, \mathcal{H}_{\lambda}\right)$. We call $\lambda$ singular if the kernel $J(\lambda)$ of the natural map $N(\lambda) \rightarrow L(\lambda)$ is non-zero and regular otherwise.

If $\left(\pi_{\lambda}, \mathcal{H}_{\lambda}\right)$ is spherical, then $L(\lambda)$ trivially is spherical, but the converse is not obvious because there is no a priori reason for an $\mathfrak{h}$-invariant linear functional on $L(\lambda)$ to extend to a continuous functional on $\mathcal{H}_{\lambda}^{\infty}$. That this is nevertheless the case follows from [2] and [4] (see also [23] for the hyperfunction version). In the light of this result we only have to deal with the question of whether $L(\lambda)$ is $\mathfrak{h}$-spherical. It is easy to see that a necessary condition is that $F(\lambda)$ is $H \cap K$ spherical. Further elementary considerations show that $N(\lambda)$ is $\mathfrak{h}$-spherical if and only if $F(\lambda)$ is $H \cap K$-spherical. This already completes the classification for all regular parameters. For singular parameters it was first observed in 23] that the condition of $F(\lambda)$ being $H \cap K$-spherical is not sufficient by showing that the even metaplectic representation of $\operatorname{Sp}(n, \mathbb{R}), n \geq 2$, is not $H$-spherical (see also 25], Cor. II.10]).

If $\lambda$ is singular, then we can attach to $L(\lambda)$ the level le $(\lambda)$ which is a natural number ranging from 1 to at most the real rank of $G$. Algebraic considerations using the fact that the maximal submodule of $N(\lambda)$ is cyclic (cf. [6]) yield the following half of the classification:

Theorem 2.8. Suppose that $L(\lambda)$ has odd reduction level. Then $\left(\pi_{\lambda}, \mathcal{H}_{\lambda}\right)$ is $H$ spherical if and only if $F(\lambda)$ is $H \cap K$-spherical.

Besides the algebraic approach to classify $H$-spherical unitary highest weight representations one can also attack the problem in an analytic way by using a realization of $\left(\pi_{\lambda}, \mathcal{H}_{\lambda}\right)$, where the $H$-action is transparent. In the sequel we assume that $G / K$ is of tube type, i.e., $G / K$ is biholomorphic to a tube domain $V+i \Omega$ with $V$ an euclidean Jordan algebra and $\Omega \subseteq V$ the open cone of squares. Then it follows from [17] that $\mathcal{H}_{\lambda}$ can be realized as an $L^{2}$-space of $F(\lambda)$-valued functions on $\bar{\Omega}$, given by

$$
L^{2}\left(\bar{\Omega}, R_{\lambda}\right):=\left\{f: \bar{\Omega} \rightarrow F(\lambda) \text { meas. : } \int_{\bar{\Omega}}\left\langle d R_{\lambda}(x) \cdot f(x), f(x)\right\rangle<\infty\right\},
$$

where $R_{\lambda}$ is a certain measure on $\bar{\Omega}$ with values in the cone $\operatorname{Herm}^{+}(F(\lambda))$ of positive operators on $F(\lambda)$. We call $L^{2}\left(\bar{\Omega}, R_{\lambda}\right)$ the cone realization of $\left(\pi_{\lambda}, \mathcal{H}_{\lambda}\right)$. The $H$ action is then given by

$$
\left.\left(\pi_{\lambda}(h) . f\right)(x)=\tau_{\lambda}(\theta(h)) . f\left(\theta(h)^{-1} . x\right) \quad \text { for } \quad h \in H, f \in L^{2}\left(\bar{\Omega}, R_{\lambda}\right)\right),
$$

where $\tau_{\lambda}$ denotes the natural $H$-representation on $F(\lambda)$ associated to the corresponding representation of $K_{\mathbb{C}}$ by a Cayley transform mapping $H$ into $K_{\mathbb{C}}$. The $K$-action however is completely invisible in the cone realization $L^{2}\left(\bar{\Omega}, R_{\lambda}\right)$. Therefore the following description of the $K$-finite vectors is surprisingly explicit and simple:

Theorem 4.5. The $K$-finite vectors in $L^{2}\left(\bar{\Omega}, R_{\lambda}\right)$ are given by $\left.e^{-\operatorname{tr}} \operatorname{Pol}(V, F(\lambda))\right|_{\bar{\Omega}}$, where tr denotes the Jordan algebra trace of $V$.

We even obtain a natural identification of $N(\lambda)$ with the space $e^{-\operatorname{tr}} \operatorname{Pol}(V, F(\lambda))$, such that the restriction map to $L^{2}\left(\bar{\Omega}, R_{\lambda}\right)$ corresponds to the quotient map $N(\lambda) \rightarrow$ 
$L(\lambda)$. Using Theorem 4.5 and the results of [17], we obtain a description of the (up to scalar multiples) unique $H$-invariant $F(\lambda)$-valued tempered distribution $R_{\lambda}^{H}$ supported by $\bar{\Omega}$. Now $L(\lambda)$ is spherical if and only if $R_{\lambda}^{H}$ vanishes on the maximal submodule $J(\lambda)$ of $N(\lambda)$, realized as $e^{-\operatorname{tr}} \operatorname{Pol}(V, F(\lambda))$. This requirement leads to the necessary condition $\operatorname{supp}\left(R_{\lambda}^{H}\right) \subseteq \operatorname{supp}\left(R_{\lambda}\right)$, but unfortunately this condition is not sufficient for $L(\lambda)$ to be spherical. Nevertheless, if $\operatorname{supp}\left(R_{\lambda}\right)$ is strictly smaller than $\bar{\Omega}$, then the information on $J(\lambda)$ and $R_{\lambda}^{H}$ is explicit enough to obtain the following Classification Theorem:

Theorem 5.11. Let $G$ be a simply connected hermitian Lie group of tube type and $\left(\pi_{\lambda}, \mathcal{H}_{\lambda}\right)$ be a unitary highest weight representation with $F(\lambda)^{H \cap K} \neq\{0\}$ and $\operatorname{supp}\left(R_{\lambda}\right) \neq \bar{\Omega}$. Then $\left(\pi_{\lambda}, \mathcal{H}_{\lambda}\right)$ is $H$-spherical if and only if $\operatorname{le}(\lambda)$ is odd.

We will see in Section 5 that among the singular unitary highest weight representations with spherical $F(\lambda)$ the condition that $\operatorname{supp}\left(R_{\lambda}\right)$ is smaller than $\bar{\Omega}$ is satisfied in about half of all cases. In the scalar case $F(\lambda)$ is always $H \cap K$-spherical and $\lambda$ is singular if and only $\operatorname{supp}\left(R_{\lambda}\right) \neq \bar{\Omega}$. Therefore the Classification Theorem covers in particular the scalar case. It therefore implies that the most singular scalar type highest weight representation which has reduction level 2 is never spherical. This covers the even metaplectic representation of $G=\operatorname{Sp}(n, \mathbb{R})$.

In the vector case (i.e. $\operatorname{dim} F(\lambda)>1$ ) the situation is much more complicated if $\operatorname{supp}\left(R_{\lambda}\right)=\bar{\Omega}$ and $\lambda$ is singular. Here we do not know of any example with even reduction level, where $L(\lambda)$ is spherical.

We conclude the paper with an appendix where we give a simple algebraic proof of the fact that parameters $\lambda$ which belong to the relative holomorphic discrete series representations of $G / H$ are always regular. This means that these representations do not provide any further information on the question of whether a singular unitary highest weight representation $L(\lambda)$ with spherical highest $K$-type $F(\lambda)$ is spherical or not.

\section{Generalities on highest Weight modules}

In this section we recall some concepts and results related to spherical highest weight modules.

Hermitian Lie algebras. Let $\mathfrak{g}$ be a simple real Lie algebra and $\theta$ a Cartan involution of $\mathfrak{g}$ with Cartan decomposition $\mathfrak{g}=\mathfrak{k} \oplus \mathfrak{p}$. We call $\mathfrak{g}$ hermitian if $\mathfrak{z}(\mathfrak{k}) \neq\{0\}$. We now collect some basic facts concerning hermitian Lie algebras (cf. [14, Ch. VIII]). The center of $\mathfrak{k}$ is one-dimensional, i.e., $\mathfrak{z}(\mathfrak{k})=\mathbb{R} i Z_{0}$ for some $0 \neq Z_{0} \in i_{\mathfrak{z}}(\mathfrak{k})$. We can normalize $Z_{0}$ such that $\operatorname{Spec}\left(Z_{0}\right)=\{1,0,-1\}$. Further, every Cartan subalgebra $\mathfrak{t}$ of $\mathfrak{k}$ is a Cartan subalgebra of $\mathfrak{g}$ and $\mathfrak{z}(\mathfrak{k}) \subseteq \mathfrak{t}$. Let $\mathfrak{g}_{\mathbb{C}}$ be the complexification of $\mathfrak{g}$ and $\widehat{\Delta}$ the root system of $\mathfrak{g}_{\mathbb{C}}$ with respect to $\mathfrak{t}_{\mathbb{C}}$.

A root $\widehat{\alpha} \in \widehat{\Delta}$ is called compact if $\widehat{\alpha}\left(Z_{0}\right)=0$ and non-compact otherwise. We denote by $\widehat{\Delta}_{k}$, resp. $\widehat{\Delta}_{n}$, the set of compact, resp. non-compact roots. We fix a positive system $\widehat{\Delta}^{+} \subseteq \widehat{\Delta}$ such that

$$
\widehat{\Delta}_{n}^{+}:=\widehat{\Delta}_{n} \cap \widehat{\Delta}^{+}=\left\{\widehat{\alpha} \in \widehat{\Delta}_{n}: \widehat{\alpha}\left(Z_{0}\right)=1\right\} .
$$

We set $\mathfrak{p}^{ \pm}:=\left\{X \in \mathfrak{g}_{\mathbb{C}}:\left[Z_{0}, X\right]= \pm X\right\}$ and note that

$$
\mathfrak{g}_{\mathbb{C}}=\mathfrak{p}^{+} \oplus \mathfrak{k}_{\mathbb{C}} \oplus \mathfrak{p}^{-}
$$


As $\operatorname{Spec}\left(Z_{0}\right)=\{1,0,-1\}$ and $Z_{0} \in \mathfrak{z}\left(\mathfrak{k}_{\mathbb{C}}\right)$, it follows that $\left[\mathfrak{k}_{\mathbb{C}}, \mathfrak{p}^{ \pm}\right] \subseteq \mathfrak{p}^{ \pm},\left[\mathfrak{p}^{+}, \mathfrak{p}^{-}\right] \subseteq$ $\mathfrak{k}_{\mathbb{C}},\left[\mathfrak{p}^{+}, \mathfrak{p}^{+}\right]=\{0\}$ and $\left[\mathfrak{p}^{-}, \mathfrak{p}^{-}\right]=\{0\}$.

Generalized Verma modules. In this subsection we collect some basic facts concerning highest weight modules from an abstract algebraic point of view. References for the fact used in this subsection are 9] or 26, Ch. IX].

Let $X \mapsto \bar{X}$ denote the conjugation in $\mathfrak{g}_{\mathbb{C}}$ with respect to the real form $\mathfrak{g}$. Then the map $X \mapsto X^{*}:=-\bar{X}$ extends to an involutive antilinear antiautomorphism

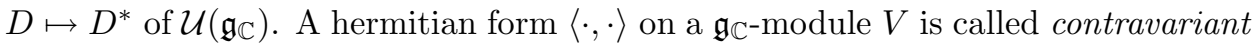
if

$$
\left(\forall X \in \mathfrak{g}_{\mathbb{C}}\right)(\forall v, w \in V) \quad\langle X . v, w\rangle=\left\langle v, X^{*} . w\right\rangle .
$$

Let $\lambda \in i t^{*}$ be dominant integral with respect to $\widehat{\Delta}_{k}^{+}$and write $F(\lambda)$ for the corresponding finite-dimensional unitary irreducible $\mathfrak{k}_{\mathbb{C}}$-module. Let $\mathfrak{q}:=\mathfrak{p}^{+} \rtimes \mathfrak{k}_{\mathbb{C}}$ and turn $F(\lambda)$ into a $\mathfrak{q}$-module by letting $\mathfrak{p}^{+}$act trivially. We define the generalized Verma module associated to $\lambda$ by

$$
N(\lambda)=\mathcal{U}\left(\mathfrak{g}_{\mathbb{C}}\right) \otimes_{\mathcal{U}(\mathfrak{q})} F(\lambda) .
$$

Then $N(\lambda)$ is a highest weight module with respect to $\widehat{\Delta}^{+}$and highest weight $\lambda$ which contains $F(\lambda)$ as a $\mathfrak{k}$-submodule which is called the highest $\mathfrak{k}$-type. Therefore it has a unique maximal submodule $J(\lambda)$ and hence a unique simple quotient $L(\lambda):=$ $N(\lambda) / J(\lambda)$. The positive definite form on $F(\lambda)$ has a unique extension to $N(\lambda)$ called the Shapovalov form. The radical of this form is the maximal submodule $J(\lambda)$. In particular, the Shapovalov form factors to a contravariant form on $L(\lambda)$ which we also denote by $\langle\cdot, \cdot\rangle_{\lambda}$. We call $L(\lambda)$ unitarizable if $\langle\cdot, \cdot\rangle_{\lambda}$ is positive definite on $L(\lambda)$.

The canonical mapping $\mathcal{U}\left(\mathfrak{p}^{-}\right) \otimes F(\lambda) \rightarrow N(\lambda)$ gives rise to an isomorphism

$$
\mathcal{U}\left(\mathfrak{p}^{-}\right) \otimes F(\lambda) \cong N(\lambda)
$$

of $\mathfrak{k}_{\mathbb{C}} \ltimes \mathfrak{p}^{-}$-modules, where $\mathfrak{k}_{\mathbb{C}} \ltimes \mathfrak{p}^{-}$acts on $\mathcal{U}\left(\mathfrak{p}^{-}\right) \otimes F(\lambda)$ via

$$
\begin{array}{ll}
\left(\forall X \in \mathfrak{k}_{\mathbb{C}}\right) & X .(p \otimes v):=X . p \otimes v+p \otimes X . v, \\
\left(\forall Y \in \mathfrak{p}^{-}\right) & Y .(p \otimes v):=Y p \otimes v
\end{array}
$$

for all $p \in \mathcal{U}\left(\mathfrak{p}^{-}\right)$and $v \in F(\lambda)$.

Symmetric structures. We now endow $\mathfrak{g}$ with a symmetric structure, i.e., with an involutive automorphism $\tau: \mathfrak{g} \rightarrow \mathfrak{g}$. The pair $(\mathfrak{g}, \tau)$ is called a symmetric Lie algebra. We write

$$
\mathfrak{h}:=\{X \in \mathfrak{g}: \tau(X)=X\} \quad \text { and } \quad \mathfrak{q}:=\{X \in \mathfrak{g}: \tau(X)=-X\}
$$

for the $\tau$-eigenspaces and note that $\mathfrak{g}=\mathfrak{h} \oplus \mathfrak{q}$. We assume that $\tau$ commutes with the Cartan involution $\theta$.

Definition 1.1. A hermitian symmetric Lie algebra $(\mathfrak{g}, \tau)$ is called compactly causal if $\mathfrak{z}(\mathfrak{k}) \subseteq \mathfrak{q}$.

Remark 1.2. For later reference we collect the basic structural facts concerning compactly causal symmetric Lie algebras. Let $(\mathfrak{g}, \tau)$ be compactly causal. We denote the complex linear extensions of $\tau$ and $\theta$ to $\mathfrak{g}_{\mathbb{C}}$ by the same symbols. The $c$-dual $\left(\mathfrak{g}^{c}, \tau^{c}\right)$ of $(\mathfrak{g}, \tau)$ is defined by $\mathfrak{g}^{c}:=\mathfrak{h}+i \mathfrak{q}$ and $\tau^{c}:=\left.\tau\right|_{\mathfrak{g}^{c}}$. Then $\theta^{c}:=\left.\theta \tau\right|_{\mathfrak{g}^{c}}$ 
defines a Cartan involution on $\mathfrak{g}^{c}$ and we write $\mathfrak{g}^{c}=\mathfrak{k}^{c} \oplus \mathfrak{p}^{c}$ for the corresponding Cartan decomposition.

As $(\mathfrak{g}, \tau)$ is compactly causal, we find a maximal abelian subspace $\mathfrak{a} \subseteq(i \mathfrak{q}) \cap \mathfrak{p}^{c}$ which is already maximal abelian in $\mathfrak{p}^{c}$ (cf. [18, Prop. 3.1.11]). We write $\Delta=$ $\Delta\left(\mathfrak{g}^{c}, \mathfrak{a}\right)$ for the restricted root system of $\mathfrak{g}^{c}$ with respect to $\mathfrak{a}$ and write

$$
\mathfrak{g}^{c}=\mathfrak{a} \oplus \mathfrak{z} \mathfrak{h}(\mathfrak{a}) \oplus \bigoplus_{\alpha \in \Delta}\left(\mathfrak{g}^{c}\right)^{\alpha}
$$

for the corresponding root space decomposition. We now choose the compact Cartan subalgebra $\mathfrak{t}$ of $\mathfrak{g}$ such that $\mathfrak{t}=(\mathfrak{t} \cap \mathfrak{h}) \oplus i \mathfrak{a}$. Since $\mathfrak{z}(\mathfrak{k}) \subseteq \mathfrak{q}$ we can choose $\widehat{\Delta}^{+}$ such that the prescription $\Delta^{+}:=\left.\widehat{\Delta}^{+}\right|_{\mathfrak{a}} \backslash\{0\}$ defines a positive system $\Delta^{+}$of $\Delta$. Similarly we define compact and non-compact roots $\Delta_{k}$ and $\Delta_{n}$. Finally we define subalgebras

$$
\mathfrak{n}^{+}:=\bigoplus_{\alpha \in \Delta^{+}}\left(\mathfrak{g}^{c}\right)^{\alpha} \quad \text { and } \quad \mathfrak{n}^{-}:=\bigoplus_{\alpha \in-\Delta^{+}}\left(\mathfrak{g}^{c}\right)^{\alpha} .
$$

Note that $\mathfrak{g}^{c}=\mathfrak{h} \oplus \mathfrak{a} \oplus \mathfrak{n}^{+}$and $\mathfrak{n}^{ \pm}=\mathfrak{p}^{ \pm} \cap \mathfrak{g}^{c}$.

\section{Spherical highest weight modules.}

Definition 1.3. (a) If $\mathfrak{h}$ is a Lie algebra and $V$ a complex $\mathfrak{h}$-module, then we write $V^{\mathfrak{h}}$ for the submodule of $V$ which is annihilated by $\mathfrak{h}$. We denote by $V^{\sharp}$ the algebraic antidual of $V$, i.e., the space of antilinear functionals $V \rightarrow \mathbb{C}$.

(b) If $(\mathfrak{g}, \tau)$ is a hermitian symmetric Lie algebra and $L(\lambda)$ an irreducible highest weight module, then we call $L(\lambda)$ spherical if there exists $0 \neq \nu \in L(\lambda)^{\sharp}$ which is annihilated by $\mathfrak{h}$, i.e., $\left(L(\lambda)^{\sharp}\right)^{\mathfrak{h}} \neq\{0\}$.

Proposition 1.4. If the hermitian symmetric Lie algebra $(\mathfrak{g}, \tau)$ has a non-trivial spherical unitarizable highest weight module, then $(\mathfrak{g}, \tau)$ is compactly causal.

Proof. [24, Lemma B.1].

Proposition 1.5. Let $V$ be a highest weight module of the compactly causal symmetric Lie algebra $(\mathfrak{g}, \tau)$ with highest weight $\lambda$ and let $v_{\lambda}$ denote a highest weight vector. Then

$$
\mathcal{U}\left(\mathfrak{h}_{\mathbb{C}}\right) \cdot v_{\lambda}=V .
$$

If, in addition, $V$ is spherical, then the highest $\mathfrak{k}_{\mathbb{C}}$-type $F(\lambda):=\left\{v \in V: \mathfrak{p}^{+} . v=\right.$ $\{0\}\}$ of $V$ is spherical for $(\mathfrak{k}, \tau)$, i.e., $F(\lambda)^{\mathfrak{h} \cap \mathfrak{k}} \neq\{0\}$.

Proof. Since $(\mathfrak{g}, \tau)$ is compactly causal, the decomposition $\mathfrak{g}^{c}=\mathfrak{h} \oplus \mathfrak{a} \oplus \mathfrak{n}^{+}$implies that $\mathfrak{g}_{\mathbb{C}}=\mathfrak{h}_{\mathbb{C}} \oplus \mathfrak{a}_{\mathbb{C}} \oplus \mathfrak{n}_{\mathbb{C}}^{+}$. Therefore $\mathcal{U}\left(\mathfrak{g}_{\mathbb{C}}\right)=\mathcal{U}\left(\mathfrak{h}_{\mathbb{C}}\right) \mathcal{U}\left(\mathfrak{a}_{\mathbb{C}}\right) \mathcal{U}\left(\mathfrak{n}_{\mathbb{C}}^{+}\right)$by the PoincaréBirkhoff-Witt Theorem, and therefore

$$
V=\mathcal{U}\left(\mathfrak{g}_{\mathbb{C}}\right) \cdot v_{\lambda}=\mathcal{U}\left(\mathfrak{h}_{\mathbb{C}}\right) \mathcal{U}\left(\mathfrak{a}_{\mathbb{C}}\right) \mathcal{U}\left(\mathfrak{n}_{\mathbb{C}}^{+}\right) \cdot v_{\lambda}=\mathcal{U}\left(\mathfrak{h}_{\mathbb{C}}\right) \cdot v_{\lambda}
$$

Now we assume that $V$ is spherical and consider $\nu \in\left(V^{\sharp}\right)^{\mathfrak{h}} \backslash\{0\}$. Then the first part implies that $\left\langle\nu, v_{\lambda}\right\rangle \neq 0$. Thus $\left.\nu\right|_{F(\lambda)}$ defines a non-zero $\mathfrak{h} \cap \mathfrak{k}$-fixed element.

The following lemma provides some refined information on the $\mathfrak{h}$-module structure of $N(\lambda)$.

Lemma 1.6. If $(\mathfrak{g}, \tau)$ is compactly causal, then we have an isomorphism of $\mathcal{U}\left(\mathfrak{h}_{\mathbb{C}}\right)$ modules:

$$
\mathcal{U}\left(\mathfrak{h}_{\mathbb{C}}\right) \otimes_{\mathcal{U}\left(\mathfrak{h}_{\mathbb{C}} \cap \mathfrak{k}_{\mathbb{C}}\right)} F(\lambda) \rightarrow N(\lambda)=\mathcal{U}\left(\mathfrak{g}_{\mathbb{C}}\right) \otimes_{\mathcal{U}\left(\mathfrak{k}_{\mathbb{C}} \oplus \mathfrak{p}^{+}\right)} F(\lambda), \quad u \otimes v \mapsto u \otimes v .
$$


Proof. [16, Lemma 3.1.1].

Proposition 1.7. If $(\mathfrak{g}, \tau)$ is compactly causal, then the restriction map

$$
\left(N(\lambda)^{\sharp}\right)^{\mathfrak{h}} \rightarrow\left(F(\lambda)^{\sharp}\right)^{\mathfrak{h} \cap \mathfrak{k}},\left.\quad \nu \mapsto \nu\right|_{F(\lambda)}
$$

is a bijection. In particular, $\operatorname{dim}\left(N(\lambda)^{\sharp}\right)^{\mathfrak{h}} \leq 1$ and $N(\lambda)$ is spherical if and only if $F(\lambda)$ is. If $F(\lambda)$ is spherical, then $L(\lambda)$ is spherical if and only a non-zero element $\nu \in\left(N(\lambda)^{\sharp}\right)^{\mathfrak{h}}$ vanishes on the kernel $J(\lambda)$ of the map $N(\lambda) \rightarrow L(\lambda)$.

Proof. (cf. 16, Lemma 3.1.2]) This is a direct consequence of Lemma 1.6.

\section{The ClASSIFICATION FOR ODD REDUCTION LEVELS}

We are interested in a description of those unitary highest weight modules $L(\lambda)$ which are spherical. In Proposition 1.7 we have seen that it is necessary that $F(\lambda)$ be spherical, and that this is sufficient provided that $N(\lambda) \cong L(\lambda)$. Therefore the question becomes interesting for the situations where the kernel $J(\lambda)$ of the quotient map $N(\lambda) \rightarrow L(\lambda)$ is non-trivial. In this case we will assign to $L(\lambda)$ a natural number $\leq(\lambda)$, called its reduction level. The main result of this section is that if $\leq(\lambda)$ is odd and $F(\lambda)$ is spherical, then $L(\lambda)$ is spherical. This is done by analyzing the $\mathfrak{t}_{\mathbb{C}}$-Fourier series of the spherical vector $\nu$ of $L(\lambda)$. In the following sections we will take a closer look at the cases with even reduction level.

The classification of unitarizable highest weight modules. Let $\mathfrak{g}$ be a hermitian Lie algebra, $\mathfrak{t} \subseteq \mathfrak{k}$ a Cartan subalgebra, and identify $\mathfrak{z}(\mathfrak{k})^{*}$ with the subspace $(\mathfrak{t} \cap[\mathfrak{k}, \mathfrak{k}])^{\perp} \subseteq \mathfrak{t}^{*}$. Let $\zeta \in i \mathfrak{z}(\mathfrak{k})^{*} \subseteq i \mathfrak{t}^{*}$ be normalized by $\zeta(\check{\gamma})=1$ for $\gamma \in \widehat{\Delta}_{n}^{+}$a long root. Fix $\lambda^{0} \in i \mathrm{t}^{*}$ dominant integral with respect to $\widehat{\Delta}_{k}^{+}$. For $u \in \mathbb{R}$ we set

$$
\lambda^{u}=\lambda^{0}-u \zeta .
$$

Further we define

$$
l\left(\lambda^{0}\right):=\left\{u \in \mathbb{R}: L\left(\lambda^{u}\right) \text { is unitarizable }\right\} .
$$

Theorem 2.1 (Enright-Howe-Wallach, Jakobsen). Let $\lambda^{0} \in i \mathrm{t}^{*}$ be dominant integral with respect to $\widehat{\Delta}_{k}^{+}$.

1. There exists a real number $u_{m}$ such that

$$
\left.l\left(\lambda^{0}\right)=\left\{u_{0}, \ldots, u_{m}\right\} \dot{\cup}\right] u_{m}, \infty[
$$

where $u_{j}=u_{0}+j \frac{d}{2}$ for $j=0, \ldots, m$ and $d$ is the multiplicity of the restricted roots of $\mathfrak{g}$ which are the second longest.

2. For $u \in l\left(\lambda^{0}\right)$ we have $L\left(\lambda^{u}\right) \cong N\left(\lambda^{u}\right)$ if and only if $u>u_{m}$.

Proof. 9 Th. 2.4].

The eigenspace decomposition of the symmetric algebra $\mathcal{U}\left(\mathfrak{p}^{-}\right)$with respect to the action of $Z_{0}$ by derivations yield the grading by homogeneous elements $\mathcal{U}\left(\mathfrak{p}^{-}\right)=\bigoplus_{n=0}^{\infty} \mathcal{U}\left(\mathfrak{p}^{-}\right)^{n}$. For each $n \in \mathbb{N}_{0}$ we set $N(\lambda)^{n}:=\mathcal{U}\left(\mathfrak{p}^{-}\right)^{n} \otimes F(\lambda)$. Then $N(\lambda)=\bigoplus_{n=0}^{\infty} N(\lambda)^{n}$ as $\mathfrak{k}_{\mathbb{C}}$-modules, and (1.1) shows that $Z_{0}$ acts on $N(\lambda)^{n}$ by multiplication with $\lambda\left(Z_{0}\right)-n$. 
Definition 2.2. Suppose that $\lambda \in i \mathfrak{t}^{*}$ is dominant integral with respect to $\widehat{\Delta}_{k}^{+}$and let $J(\lambda)$ be the maximal submodule of $N(\lambda)$. We call $\lambda$ regular if $J(\lambda)=\{0\}$ and singular otherwise. Let $J(\lambda)=\bigoplus_{n=0}^{\infty} J(\lambda)^{n}$ be the natural grading induced from the one on $N(\lambda)$. If $\lambda$ is singular, then we call the number

$$
\operatorname{le}(\lambda):=\min \left\{n \in \mathbb{N}_{0}: J(\lambda)^{n} \neq\{0\}\right\}
$$

the level of reduction of $L(\lambda)$.

Remark 2.3. From the classification of unitary highest weight modules, we know that the points $u_{0}, \ldots, u_{m}$ from Theorem 2.1 are related to reduction levels by

$$
\leq\left(\lambda^{u_{j}}\right)=1+j
$$

(cf. [8]). So we see that approximately half of the singular points $u_{0}, \ldots, u_{m}$ correspond to even and half to odd reduction levels.

Theorem 2.4 (Davidson-Enright-Stanke). Suppose that $N(\lambda)$ is reducible and $L(\lambda)$ is unitarizable. Then the maximal submodule $J(\lambda)$ is a highest weight module, i.e., there exists an element $\lambda_{J} \in i \mathrm{t}^{*}$ which is dominant integral with respect to $\widehat{\Delta}_{k}^{+}$ such that $J(\lambda) \cong N\left(\lambda_{J}\right)$.

Proof. This follows from [6. Th. 3.1]. Note that, since $N(\lambda)$ is a free $\mathcal{U}\left(\mathfrak{p}^{-}\right)$-module, the maximal submodule $J(\lambda)$ is isomorphic to $N\left(\lambda_{J}\right)$ if $\lambda_{J}$ is its highest weight.

Some algebraic criteria. Let $\sigma:=\exp \left(\pi i Z_{0}\right) \in G$. Then the Cartan involution $\theta$ on $G$ is given by $\theta(g)=\sigma g \sigma^{-1}$ for $g \in G$.

Lemma 2.5 (Parity Lemma). Let $N(\lambda)$ be spherical and $\nu \in\left(N(\lambda)^{\sharp}\right)^{\mathfrak{h}}$ be a nonzero spherical vector. For $n \in \mathbb{N}_{0}$ we set $\nu^{n}:=\left.\nu\right|_{N(\lambda)^{n}}$. Then $\nu^{n}=0$ for $n$ odd.

Proof. Since the action of $\mathfrak{k}$ on $N(\lambda)$ is locally finite, it integrates a representation of the simply connected group $K \subseteq G$. Hence, in particular, $\sigma$ acts on $N(\lambda)$ in such a way that $\sigma(X . v)=\theta(X) .(\sigma . v)$ for $X \in \mathfrak{g}, v \in N(\lambda)$. Since $\mathfrak{h}$ is $\theta$-invariant, $\nu \circ \sigma=c \nu$ for some $c \in \mathbb{C}^{\times}$follows from $\left(N(\lambda)^{\sharp}\right)^{\mathfrak{h}}=\mathbb{C} \nu$ (Proposition 1.7). From $\nu=\sum_{n=0}^{\infty} \nu^{n}$ and (1.1) we thus get

$$
\sigma . \nu=e^{\pi i \lambda\left(Z_{0}\right)} \sum_{n=0}^{\infty}(-1)^{n} \nu^{n} .
$$

Therefore $c=e^{\pi i \lambda\left(Z_{0}\right)}$ and $\nu^{n}=0$ for $n$ odd.

From now on we suppose that $(\mathfrak{g}, \tau)$ is compactly causal, that $L(\lambda)$ is unitarizable, and that $F(\lambda)$ is spherical, so that $N(\lambda)$ is spherical by Proposition 1.7. Let $0 \neq \nu \in\left(N(\lambda)^{\sharp}\right)^{\mathfrak{h}}$.

Lemma 2.6. If $J(\lambda)$ is not spherical, then $L(\lambda)$ is spherical.

Proof. If $J(\lambda)$ is not spherical, then $\left.\nu\right|_{J(\lambda)}=0$. Thus $\nu$ factors to a non-zero $\mathfrak{h}$-fixed antilinear functional on $L(\lambda)$, showing that $L(\lambda)$ is spherical.

Proposition 2.7. Let $0 \neq \nu \in\left(N(\lambda)^{\sharp}\right)^{\mathfrak{h}}$ and let $F\left(\lambda_{J}\right)$ be the highest $\mathfrak{k}$-type of $J(\lambda)$. Then the following assertions are equivalent:

1. $L(\lambda)$ is spherical.

2. $\left.\nu\right|_{F\left(\lambda_{J}\right)}=0$. 
Proof. Note that $L(\lambda)$ is spherical if and only if $\left.\nu\right|_{J(\lambda)}=0$. We recall from Theorem 2.4 that $J(\lambda)$ is a highest weight module with highest weight $\lambda_{J}$. Thus Proposition 1.5 implies that $\left.\nu\right|_{J(\lambda)}=0$ if and only if $\left.\nu\right|_{F\left(\lambda_{J}\right)}=0$.

Theorem 2.8 (Classification for odd reduction levels). If $L(\lambda) \neq N(\lambda)$ is unitarizable and $\leq(\lambda)$ is odd, then $L(\lambda)$ is spherical if and only if $F(\lambda)$ is spherical.

Proof. If $L(\lambda)$ is spherical, then $F(\lambda)$ is spherical by Proposition 1.5.

Assume, conversely, that $F(\lambda)$ is spherical. Then $N(\lambda)$ is spherical (Proposition 1.7), so that there exists a non-zero element $\nu \in\left(N(\lambda)^{\sharp}\right)^{\mathfrak{h}}$. In view of Proposition 2.7, we have to show that $\left.\nu\right|_{F\left(\lambda_{J}\right)}=0$. Since le $(\lambda)$ is odd, this follows from Lemma 2.5 .

Example 2.9. In the following we give an example where both $L(\lambda)$ and $J(\lambda)$ are spherical.

Let $(\mathfrak{g}, \mathfrak{h})=(\mathfrak{s p}(n, \mathbb{R}), \mathfrak{g l}(n, \mathbb{R}))$. We choose $\lambda^{0}=0$ and set $\lambda:=\lambda^{u_{n-1}}$ for the first reduction point which has reduction level $n$. Then it follows from 8 that the highest weight $\lambda_{J}$ of the maximal submodule $J(\lambda)$ is contained in $i_{\mathfrak{z}}(\mathfrak{k})^{*}$. In particular, $F\left(\lambda_{J}\right)$ is $\mathfrak{h} \cap \mathfrak{k}$-spherical. Since $\lambda$ is a first reduction point, we have $J(\lambda)=N\left(\lambda_{J}\right)$. Hence $J(\lambda)$ is spherical by Proposition 1.7. Further if $n$ is odd, then Theorem 2.8 implies that $L(\lambda)$ is spherical too. More details on the composition series of the modules $N(\lambda)$ in this case can be found in [10].

\section{REALizATION OF REPRESENTATIONS IN SPACES OF DistribUtions}

In this section we realize simple highest weight modules of the important special case of Cayley type compactly causal symmetric Lie algebras in spaces of vectorvalued distributions supported by a closed convex cone in a euclidean Jordan algebra. For the constructions in this section unitarizability will play no role at all. In the next section we will see how this picture can be used to get refined information on unitary highest weight modules because in this case the corresponding realization by distributions can also be viewed as a subspace of a suitable $L^{2}$-space with respect to an operator-valued measure.

\section{Algebraic preliminaries.}

Definition 3.1. A compactly causal symmetric Lie algebra $(\mathfrak{g}, \tau)$ is said to be of Cayley type if $\mathfrak{z}(\mathfrak{h}) \neq\{0\}$.

Remark 3.2. (a) If $(\mathfrak{g}, \tau)$ is of Cayley type, then $\mathfrak{z}(\mathfrak{h}) \subseteq \mathfrak{h} \cap \mathfrak{p}$ and $\operatorname{dim} \mathfrak{z}(\mathfrak{h})=1$ (cf. [18, Th. 1.3.11]).

(b) Cayley type symmetric spaces are classified (cf. [18, Th. 3.2.8]). Up to isomorphy, the corresponding pairs $(\mathfrak{g}, \mathfrak{h})$ are given by

$$
\begin{gathered}
(\mathfrak{s u}(n, n), \mathfrak{s l}(n, \mathbb{C}) \oplus \mathbb{R}), \quad\left(\mathfrak{s o}^{*}(4 n), \mathfrak{s u}^{*}(2 n) \oplus \mathbb{R}\right),(\mathfrak{s p}(n, \mathbb{R}), \mathfrak{g l}(n, \mathbb{R})), \\
(\mathfrak{s o}(2, n), \mathfrak{s o}(1, n-1) \oplus \mathbb{R}), \quad\left(\mathfrak{e}_{7(-25)}, \mathfrak{e}_{6(-26)} \oplus \mathbb{R}\right) .
\end{gathered}
$$

(c) If $(\mathfrak{g}, \tau)$ is of Cayley type, then the Lie algebra $\mathfrak{g}$ is hermitian and of tube type (cf. [18], [22]), i.e., the hermitian symmetric space $G / K$ attached to $\mathfrak{g}$ is biholomorphically equivalent to a tube domain $T_{\Omega}=V+i \Omega$ over the convex open cone $\Omega$ of invertible squares in a finite-dimensional simple euclidean Jordan algebra $V$. 
In the following $V$ denotes a simple euclidean Jordan algebra with unit element $e$ and $\Omega \subseteq V$ the open cone of invertible squares. Having Remark 3.2(b) in mind, from now on $G$ stands for the simply connected covering group of Aut $\left(T_{\Omega}\right)_{0}$ and $K=$ $\{g \in G: g . i e=i e\}$ is the analytic subgroup corresponding to $\mathfrak{k}$. The corresponding Cartan involution $\theta$ satisfies $\eta\left(g \cdot \eta^{-1}(z)\right)=\theta(g)$. $z$ for $z \in T_{\Omega}$, where $\eta(z)=-z^{-1}$. As a subgroup of $G$, the group $H$ consists of all those elements of $G$ acting by linear maps on $T_{\Omega}$. This implies in particular that $H$ preserves $V$ and also $\Omega$. Geometrically the involution $\tau$ is determined by $\zeta\left(g \cdot \zeta^{-1}(z)\right)=\tau(g) . z$ for $z \in T_{\Omega}$, where $\zeta(z)=-\bar{z}$. Since $G$ is simply connected, the subgroup $G^{\tau}$ is connected, hence coincides with $H$. Let $K_{\mathbb{C}}$ denote the universal complexification of $K$. Since $K_{\mathbb{C}}$ has a polar decomposition $K_{\mathbb{C}}=K \exp (i \mathfrak{k})$, this group is also simply connected.

Definition 3.3. (a) We follow the notation of [11]. We define $L(z) \in \operatorname{End}\left(V_{\mathbb{C}}\right)$, $z \in V_{\mathbb{C}}$, by $L(z) \cdot w=z w$ and write

$$
P: V_{\mathbb{C}} \rightarrow \operatorname{End}\left(V_{\mathbb{C}}\right), \quad z \mapsto P(z)=2 L(z)^{2}-L\left(z^{2}\right)
$$

for the the quadratic representation of $V_{\mathbb{C}}$. Let $\left\{c_{1}, \ldots, c_{r}\right\}$ be a Jordan frame of $V$ (cf. [11, p. 44]) and set $e_{k}:=c_{1}+\ldots+c_{k}$ for $0 \leq k \leq r$ with the conventions $e_{0}=0$ and $e=e_{r}$. Then $\Omega=H . e_{r}$ and

$$
\bar{\Omega}=\mathcal{O}_{0} \dot{\cup} \mathcal{O}_{1} \dot{\cup} \ldots \dot{\cup} \mathcal{O}_{r}
$$

with $\mathcal{O}_{j}=H . e_{j}$ and $\overline{\mathcal{O}_{j}}=\bigcup_{i<j} \mathcal{O}_{i}$ is the $H$-orbit decomposition of $\bar{\Omega}$ (cf. [11, Prop. IV.3.1]). The number $r$ is called the rank of the Jordan algebra $V$.

Note that $\mathfrak{h} \cap \mathfrak{k} \cong \operatorname{der}(V)$ and $\mathfrak{h} \cap \mathfrak{p} \cong\{L(x): x \in V\}$ (cf. [11, Th. III.5.1]). Then $\mathfrak{b}:=\bigoplus_{j=1}^{r} \mathbb{R} L\left(c_{j}\right)$ is a maximal abelian subspace of $\mathfrak{h} \cap \mathfrak{p}$. Define $\varepsilon_{j} \in \mathfrak{b}^{*}$ by $\varepsilon_{j}\left(L\left(c_{k}\right)\right)=\delta_{j k}$ and set $\varepsilon:=\sum_{j=1}^{r} \varepsilon_{j}$. Then the restricted root system of $\mathfrak{h}$ is given by

$$
\Sigma=\left\{\frac{1}{2}\left(\varepsilon_{j}-\varepsilon_{i}\right): i \neq j, 1 \leq i, j \leq r\right\} .
$$

We consider the positive system $\Sigma^{+}=\left\{\frac{1}{2}\left(\varepsilon_{j}-\varepsilon_{i}\right): i<j\right\}$. The coroot corresponding to $\frac{1}{2}\left(\varepsilon_{j}-\varepsilon_{i}\right)$ is $2\left(L\left(c_{j}\right)-L\left(c_{i}\right)\right)$. Therefore a linear functional $\lambda=\sum_{j=1}^{r} \frac{m_{j}}{2} \varepsilon_{j}$ is dominant integral, i.e., can occur as a restricted highest weight of a finite-dimensional representation of $\mathfrak{h}$ if and only if

$$
m_{j}-m_{i} \in \mathbb{N}_{0}, i<j,
$$

which implies in particular that $m_{1} \leq m_{2} \leq \ldots \leq m_{r}$. For real highest weight representations which are the same as spherical representations of $H$, one has the stronger condition that $m_{j}-m_{i} \in 2 \mathbb{N}_{0}$ for $i<j$ (cf. [17] App. A] and [15, Th. 5.1]).

(b) Let

$$
\mathcal{D}=\left\{z \in V_{\mathbb{C}}: I-P(z) P(\bar{z})>>0\right\} \subseteq \mathfrak{p}^{+} \cong V_{\mathbb{C}}
$$

be the Harish Chandra realization of $G / K$ as a bounded symmetric domain (cf. [11, Prop. X.4.2, Th. X.4.3]). Then the Cayley transform

$$
c: \mathcal{D} \rightarrow T_{\Omega}, \quad z \mapsto-i(z+e)(z-e)^{-1}
$$

is biholomorphic.

The universal complexification $G_{\mathbb{C}}$ of $G$ acts by meromorphic mappings on $V_{\mathbb{C}}$ and $\mathfrak{g}_{\mathbb{C}}$ can be viewed as a Lie algebra of holomorphic vector fields on $V_{\mathbb{C}}$. In this sense the Cayley transform can be considered as obtained by an element of $G_{\mathbb{C}}$, which implies that it induces an isomorphism $c_{\mathfrak{g}} \in \operatorname{Aut}\left(\mathfrak{g}_{\mathbb{C}}\right)$ mapping the Lie 
algebra $\tilde{\mathfrak{g}}$ of $\operatorname{Aut}(\mathcal{D})$ onto $\mathfrak{g}$. Note that $c_{\mathfrak{g}}=e^{i \frac{\pi}{2} \operatorname{ad} T}$ for some $\mathfrak{h} \cap \mathfrak{k}$-fixed element $T \in \mathfrak{q} \cap \mathfrak{p}$ with $\operatorname{Spec}(\operatorname{ad} T)=\{-1,0,1\}$ (cf. [18]). The subalgebra $\widetilde{\mathfrak{k}} \subseteq \widetilde{\mathfrak{g}}$ consisting of all vector fields vanishing in 0 is maximal compact, and furthermore we have

$$
\widetilde{\mathfrak{k}}=(\mathfrak{h} \cap \mathfrak{k}) \oplus i(\mathfrak{h} \cap \mathfrak{p})
$$

(cf. [11, Prop. X.3.1, Th. X.5.3]). It follows in particular that $\widetilde{\mathfrak{k}}_{\mathbb{C}}=\mathfrak{h}_{\mathbb{C}}$ and that $\widetilde{\mathfrak{k}}$ is a compact real form of $\mathfrak{h}_{\mathbb{C}}$. Therefore complex $\mathfrak{h}$-modules are equivalent to complex $\widetilde{\mathfrak{k}}$-modules. In addition, we have

$$
\mathfrak{k}=c_{\mathfrak{g}}(\widetilde{\mathfrak{k}})=(\mathfrak{h} \cap \mathfrak{k}) \oplus i c_{\mathfrak{g}}(\mathfrak{h} \cap \mathfrak{p})
$$

and $i c_{\mathfrak{g}}(\mathfrak{h} \cap \mathfrak{p})=\mathfrak{q} \cap \mathfrak{k}$. We choose a compactly embedded Cartan subalgebra $\mathfrak{t} \subseteq \mathfrak{g}$ containing $i c_{\mathfrak{g}}(\mathfrak{b})$, which is then automatically adapted to the above decomposition of $\mathfrak{k}$. We choose a positive system $\widehat{\Delta}_{k}^{+}$of the corresponding root system $\widehat{\Delta}_{k}=$ $\Delta\left(\mathfrak{k}_{\mathbb{C}}, \mathfrak{t}_{\mathbb{C}}\right)$ such that $\left.\widehat{\Delta}_{k}^{+} \circ c_{\mathfrak{g}}\right|_{\mathfrak{b}} \subseteq \Sigma^{+} \cup\{0\}$.

If $\left(\pi_{\lambda}^{\mathfrak{k}}, F(\lambda)\right)$ is a highest weight representation of $\mathfrak{k}$, resp. $\mathfrak{k}_{\mathbb{C}}$, we obtain a representation of $\mathfrak{h}_{\mathbb{C}}=\widetilde{\mathfrak{k}}_{\mathbb{C}}$ by $\tau_{\lambda}^{\mathfrak{h}}:=\pi_{\lambda}^{\mathfrak{k}} \circ c_{\mathfrak{g}}$. We call $\left.\lambda \circ c_{\mathfrak{g}}\right|_{\mathfrak{b}}$ the restricted highest weight of this representation (cf. (a)).

On the group level the inclusion $c_{\mathfrak{g}}^{-1}: \mathfrak{h} \rightarrow \mathfrak{k}_{\mathbb{C}}$ yields the identity on $H \cap K$. Therefore the Cartan decomposition $H=(H \cap K) \exp (\mathfrak{h} \cap \mathfrak{p})$ leads to a homomorphism $H \rightarrow K_{\mathbb{C}}$ which, in view of the simple connectedness of $K_{\mathbb{C}}$, is the universal complexification of $H$, i.e., $H_{\mathbb{C}} \cong K_{\mathbb{C}}$.

Polynomial differential operators and distributions. For a finite-dimensional complex Hilbert vector space $F$, let $\mathrm{SO}(F)$ denote the space of $F$-valued tempered distributions on $V$ supported by the closed convex cone $\bar{\Omega}$. For $z \in V_{\mathbb{C}}$ let $e_{z}$ : $V \rightarrow \mathbb{C}$ be defined by $e_{z}(x):=e^{(z, x)}$, where $(\cdot, \cdot): V_{\mathbb{C}} \times V_{\mathbb{C}} \rightarrow \mathbb{C}$ is the complex bilinear extension of the scalar product on $V$. According to Lemma B.1 in [17, for each $z \in T_{\Omega}$ and $D \in \mathrm{SO}(F)$ we have $e_{i z} D \in \mathrm{SO}(F)$ and $\left.e_{i z}\right|_{\Omega}$ extends to a Schwartz function on $V$, so that the Fourier transform $\mathcal{F}(D)$ extends uniquely to a holomorphic function

$$
\mathcal{F}(D): T_{\Omega} \rightarrow F, \quad z \mapsto D\left(e_{i z}\right)
$$

(cf. [31, Ch. VIII, Prop. 6]). We thus obtain an injective map

$$
\mathcal{F}: \mathrm{SO}(F) \rightarrow \operatorname{Hol}\left(T_{\Omega}, F\right) .
$$

For a characterization of the image we refer to [31, Ch. VIII]. Here we will only need that this map is injective.

In the following we will consider the space $\mathcal{S}^{\prime}(V, F)=\mathcal{S}^{\prime}(V) \otimes V$ of $F$-valued tempered distributions as the topological antidual space of $\mathcal{S}(V, F)$, the $F$-valued Schwartz space, where the pairing is given by

$$
\langle D \otimes v, \varphi \otimes w\rangle:=\overline{D(\varphi)}\langle v, w\rangle .
$$

Now we turn to the action of the algebra of polynomial differential operators on these spaces. To fix the notation, for $v \in V$ we define on $C^{\infty}(V, F)$ the operators

$$
\left(\partial_{v} \cdot \varphi\right)(x)=d \varphi(x)(v) \quad \text { and } \quad\left(m_{v} \cdot \varphi\right)(x)=(v, x) \varphi(x) .
$$

It is also clear that $\operatorname{End}(F)$ acts naturally on $C^{\infty}(V, F)$ by $A . \varphi:=A \circ \varphi$. We write $\mathcal{P} \mathcal{D}(V, F)$ for the algebra generated by the operators $\partial_{v}, m_{v}$ and $\operatorname{End}(F)$. The elements of this algebra are sums of operators of the type $P(x) \partial_{v_{1}} \cdots \partial_{v_{n}}$, where 
$P: V \rightarrow \operatorname{End}(F)$ is a polynomial function. We define an antilinear involution $*$ on $\mathcal{P} \mathcal{D}(V, F)$ by prescribing on the generators that

$$
m_{v}^{*}:=m_{v}, \quad \partial_{v}^{*}:=-\partial_{v} \quad \text { and } \quad A^{*}:=A^{*},
$$

where $A^{*}$ for $A \in \operatorname{End}(F)$ denotes the operator adjoint. Then we obtain a natural action of $\mathcal{P} \mathcal{D}(V, F)$ on $\mathrm{SO}(F)$ by

$$
\langle P . D, \varphi\rangle:=\left\langle D, P^{*} \cdot \varphi\right\rangle .
$$

Here we use that the elements of $\mathcal{P} \mathcal{D}(V, F)$ restrict to continuous operators on the Schwartz space $\mathcal{S}(V, F)$. In particular we have

$$
\left\langle\partial_{v} . D, \varphi\right\rangle=\left\langle D,-\partial_{v} \cdot \varphi\right\rangle \quad \text { and } \quad\left\langle m_{v} . D, \varphi\right\rangle=\left\langle D, m_{v} \cdot \varphi\right\rangle .
$$

It is also clear how the algebra $\mathcal{P} \mathcal{D}(V, F)$ acts on the space $\operatorname{Hol}\left(T_{\Omega}, F\right)$. In this sense we obtain directly from the definitions:

$$
\mathcal{F}\left(\partial_{v} . D\right)=-i m_{v} \cdot \mathcal{F}(D), \quad \mathcal{F}\left(m_{v} . D\right)=-i \partial_{v} . \mathcal{F}(D), \quad \mathcal{F}(A . D)=A . \mathcal{F}(D)
$$

for $v \in V$ and $A \in \operatorname{End}(F)$. The preceding relations describe in which way the natural action of the algebra $\mathcal{P} \mathcal{D}(V, F)$ on $\mathrm{SO}(F)$ is intertwined with its natural action on the space $\operatorname{Hol}\left(T_{\Omega}, V\right)$. The Fourier transform defines an algebra automorphism $\mathcal{F}: \mathcal{P D}(V, F) \rightarrow \mathcal{P} \mathcal{D}(V, F)$ which satisfies for all $P \in \mathcal{P} \mathcal{D}(V, F)$ and $D \in \mathcal{S}^{\prime}(V, F)$

$$
\mathcal{F}(P . D)=\mathcal{F}(P) \mathcal{F}(D) .
$$

Now (3.1) can be written as

$$
\mathcal{F}\left(\partial_{v}\right)=-i m_{v}, \quad \mathcal{F}\left(m_{v}\right)=-i \partial_{v} \quad \text { and } \quad \mathcal{F}(A)=A .
$$

Lie algebra actions. Recall that we consider $G$ as the universal covering group of $\operatorname{Aut}\left(T_{\Omega}\right)_{0}$. We write $\operatorname{Str}\left(V_{\mathbb{C}}\right)$ for the structure group of the complex Jordan algebra $V_{\mathbb{C}}$ (cf. [11, p. 147]). Then the natural homomorphism $H \rightarrow \operatorname{Str}\left(V_{\mathbb{C}}\right)$, coming from the inclusion $H \subseteq G$, induces a holomorphic covering $H_{\mathbb{C}} \rightarrow \operatorname{Str}\left(V_{\mathbb{C}}\right)_{0}$, where $H_{\mathbb{C}}$ denotes the universal Lie group complexification of $H$. The group $G$ naturally acts on $T_{\Omega}$ and

$$
J: G \times T_{\Omega} \rightarrow \operatorname{Str}\left(V_{\mathbb{C}}\right)_{0}, \quad(g, z) \mapsto J(g, z):=\frac{\partial(g . z)}{\partial z}
$$

defines a cocycle (cf. 11, Prop. XIII.4.1]). Using the simply connectedness of $T_{\Omega}$, it is not hard to see that there is a unique lifting of $J$ to a cocycle

$$
\widetilde{J}: G \times T_{\Omega} \rightarrow H_{\mathbb{C}} \quad \text { with } \quad \widetilde{J}(\mathbf{1}, z)=\mathbf{1} .
$$

Let $(\tau, F)$ be a holomorphic representation of $H_{\mathbb{C}}$. Then we obtain by

$$
J_{\tau}: G \times T_{\Omega} \rightarrow \mathrm{GL}(F), \quad(g, z) \mapsto J_{\tau}(g, z):=\tau(\widetilde{J}(g, z))
$$

a $\operatorname{GL}(F)$-valued cocycle which leads to a representation of $G$ on $\operatorname{Hol}\left(T_{\Omega}, F\right)$ given by

$$
\left(\pi_{\tau}(g) . f\right)(z)=J_{\tau}\left(g^{-1}, z\right)^{-1} \cdot f\left(g^{-1} \cdot z\right) \quad \text { for } \quad g \in G, f \in \operatorname{Hol}\left(T_{\Omega}, F\right) .
$$

For $v \in V$ and $h \in H$ we obtain in particular the simple formulas

$$
\left(\pi_{\tau}(v) . f\right)(z)=f(z-v) \quad \text { and } \quad\left(\pi_{\tau}(h) \cdot f\right)(z)=\tau(h) \cdot f\left(h^{-1} \cdot z\right) .
$$

The derived action $d \pi_{\tau}$ of the Lie algebra $\mathfrak{g}$ on $\operatorname{Hol}\left(T_{\Omega}, F\right)$ is then given by

$$
\left(d \pi_{\tau}(X) \cdot f\right)(z)=d_{1} J_{\tau}(\mathbf{1}, z)(X) \cdot f(z)+(\dot{\sigma}(X) \cdot f)(z),
$$


where $\dot{\sigma}(X)(z):=\left.\frac{d}{d t}\right|_{t=0} \exp (-t X) . z$ is the holomorphic vector field on $T_{\Omega}$ corresponding to the action of the one-parameter subgroup $\exp (\mathbb{R} X) \subseteq G$ (cf. [26] Prop. IV.1.9(ii)]).

Lemma 3.4. The action of $\mathfrak{g}$ on $\operatorname{Hol}\left(T_{\Omega}, F\right)$ is given explicitly by polynomial differential operators as follows:

1. $(v \cdot f)(z)=-d f(z)(v)$ for $v \in V \cong \mathfrak{n}^{+}$,

2. $(X . f)(z)=d \tau(X) . f(z)-d f(z)(X . z)$ for $X \in \mathfrak{h}$, and

3. $(\theta(v) . f)(z)=2 d \tau(z \square v) . f(z)-d f(z)(P(z) . v)$ for $v \in V$, where $z \square v \in \mathfrak{s t r}\left(V_{\mathbb{C}}\right)$ $\cong \mathfrak{h}_{\mathbb{C}}$ is defined by $a \square b:=L(a b)+[L(a), L(b)]$.

Proof. In this proof we assume that $v \in V, X \in \mathfrak{h}$ and $h \in H$. According to 11 p. 209], the vector fields corresponding to the action of $\mathfrak{g}$ on $T_{\Omega}$ are given by

$$
\dot{\sigma}(v)(z)=-v, \quad \dot{\sigma}(X)(z)=-X . z \quad \text { and } \quad \dot{\sigma}(\theta . v)(z)=-P(z) \cdot v,
$$

where the last assertion follows from the fact that the inverion $\eta: T_{\Omega} \rightarrow T_{\Omega}, \eta(z)=$ $-z^{-1}$ satisfies $\eta(g . z)=\theta(g) . \eta(z)$ for $g \in G$ and $z \in T_{\Omega}$.

We further have

$$
J_{\tau}(v, z)=\mathbf{1}, \quad J_{\tau}(h, z)=\tau(h) \quad \text { and } \quad J_{\tau}(\theta . v, z)=\tau\left(\widetilde{P}\left(v-z^{-1}\right)^{-1} \widetilde{P}(z)^{-1}\right) .
$$

Here the first two formulas are clear from the definitions, and the last one follows from $\theta(v) . z=\eta(v+\eta(z)), d \eta(z)=P(z)^{-1}$ (11, Prop. II.3.3]) and the chain rule. This leads to $d_{1} J_{\tau}(\mathbf{1}, z) \cdot v=0$ and $d_{1} J_{\tau}(\mathbf{1}, z) \cdot X=d \tau(X)$, so that it only remains to verify

$$
d_{1} J_{\tau}(\mathbf{1}, z) . \theta v=2 d \tau(z \square v) .
$$

We have

$$
\begin{aligned}
d_{1} J(\mathbf{1}, z) \cdot \theta v & =\left.\frac{d}{d t}\right|_{t=0} P\left(z^{-1}-t v\right)^{-1} P(z)^{-1} \\
& =-\left.P\left(z^{-1}\right)^{-1} \frac{d}{d t}\right|_{t=0} P\left(z^{-1}-t v\right) P\left(z^{-1}\right)^{-1} P(z)^{-1} \\
& =-\left.P(z) \frac{d}{d t}\right|_{t=0} P\left(z^{-1}-t v\right)=2 P(z) P\left(z^{-1}, v\right),
\end{aligned}
$$

so that the assertion follows from Lemma 3.5 below.

Lemma 3.5. For each $z \in V_{\mathbb{C}}^{\times}$and $u \in V$ we have $P(z) P\left(z^{-1}, u\right)=z \square u$.

Proof. In view of [11, p. 147], we have for all $x, y \in V_{\mathbb{C}}$ and $g \in \operatorname{Str}\left(V_{\mathbb{C}}\right)$ the identities

$$
P(g . x, g . y)=g P(x, y) g^{\top} \quad \text { and } \quad(g . x \square \theta(g) \cdot y)=g(x \square y) g^{-1} .
$$

For $z=$ g.e we thus get

$$
\begin{aligned}
P(z) P\left(z^{-1}, u\right) & =P(g . e) P\left((g . e)^{-1}, u\right)=g P(e) g^{\top} P(\theta(g) . e, u) \\
& =g g^{\top} \theta(g) P\left(e, g^{\top} . u\right) g^{-1}=g P\left(e, g^{\top} . u\right) g^{-1}=g L\left(g^{\top} . u\right) g^{-1} .
\end{aligned}
$$

On the other hand $e \square x=L(x)$ implies

$$
z \square u=(g . e) \square u=g\left(e \square g^{\top} . u\right) g^{-1}=g L\left(g^{\top} . u\right) g^{-1}=P(z) P\left(z^{-1}, u\right) .
$$

Now the assertion follows from $V_{\mathbb{C}}^{\times}=\operatorname{Str}\left(V_{\mathbb{C}}\right) . e([11$, Prop. VIII.3.5(i)]). 
The main point in the formulas of Lemma 3.4 is that they show that $d \pi_{\tau}(\mathfrak{g}) \subseteq$ $\mathcal{P} \mathcal{D}(V, F)$, acting on $\operatorname{Hol}\left(T_{\Omega}, V\right)$. We therefore obtain a representation of $\mathfrak{g}$ on $\mathrm{SO}(F)$ by

$$
X . D:=\mathcal{F}^{-1}\left(d \pi_{\tau}(X)\right) . D
$$

and further a representation $\rho_{\tau}$ on $C^{\infty}(V, F)$ which on $\mathcal{S}(V, F)$ is given by

$$
\left\langle D, \rho_{\tau}(X) . \varphi\right\rangle=\langle-X . D, \varphi\rangle \quad \text { for } \quad \varphi \in \mathcal{S}(V, F), X \in \mathfrak{g} .
$$

We denote by $\operatorname{Pol}(V, F)$ the space of polynomial mappings from $V$ to $F$.

Remark 3.6. For each linear function $\alpha \in V^{*}$ the subspace $e^{\alpha} \operatorname{Pol}(V, F) \subseteq C^{\infty}(V, F)$ is invariant under the action of $\mathfrak{g}$ because this space is invariant under the whole algebra $\mathcal{P} \mathcal{D}(V, F)$. We will see below that the most important space of this type arises for $\alpha=-\operatorname{tr}$.

The following lemma makes the action of the parabolic subalgebra $\mathfrak{n}^{+} \rtimes \mathfrak{h} \subseteq \mathfrak{g}$ quite explicit. In principle it is also possible to obtain formulas for the action of $\mathfrak{n}^{-}=\theta\left(\mathfrak{n}^{+}\right)$, but it seems that this can only be done in coordinates. Fortunately, for our arguments below it will suffice to have direct access to the action of $\mathfrak{h}$ and $\mathfrak{n}^{+}$.

Lemma 3.7. For $\varphi \in C^{\infty}(V, F)$ we have:

1. $\rho_{\tau}(v) \cdot \varphi=-i m_{v} \varphi$ for $v \in V \cong \mathfrak{n}^{+}$. In particular, $\rho_{\tau}\left(\mathcal{U}\left(\mathfrak{n}^{+}\right)\right) \cdot \varphi=\operatorname{Pol}(V) \cdot \varphi$.

2. $\left(\rho_{\tau}(X) \cdot \varphi\right)(x)=d \tau(\theta(X)) \cdot \varphi(x)-d \varphi(x) .(\theta(X) \cdot x)$ for all $X \in \mathfrak{h}$ and $x \in V$.

Proof. 1. We have $d \pi_{\tau}(v)=-\partial_{v}$ (Lemma 3.4(1)), hence $\mathcal{F}^{-1}\left(d \pi_{\tau}(v)\right)=-i m_{v}$, and therefore

$$
\left\langle D, \rho_{\tau}(v) \cdot \varphi\right\rangle=-\left\langle\left(-i m_{v}\right) \cdot D, \varphi\right\rangle=\left\langle i m_{v} \cdot D, \varphi\right\rangle=\left\langle D,-i m_{v} \varphi\right\rangle
$$

because the pairing $\langle\cdot, \cdot\rangle$ is sesquilinear.

2. Let $v_{1}, \ldots, v_{n}$ be a basis of $V, m_{j}:=m_{v_{j}}$, and $\partial_{j}:=\partial_{v_{j}}$. Then the differential operator on $\operatorname{Hol}\left(T_{\Omega}, F\right)$ corresponding to $X \in \mathfrak{h}$ is given by

$$
\left(d \pi_{\tau}(X) . f\right)(z)=d \tau(X) . f(z)-d f(z)(X . z)=d \tau(X) . f(z)-\left(\sum_{j, k=1}^{n} x_{j k} m_{k} \partial_{j} \cdot f\right)(z)
$$

where $X . v_{j}=\sum_{k=1}^{n} x_{k j} v_{k}$. The map $\mathcal{F}^{-1}$ maps this operator to

$$
d \tau(X)-\sum_{j, k=1}^{n} x_{j k} i \partial_{k} i m_{j}=d \tau(X)+\sum_{j, k=1}^{n} x_{j k} \partial_{k} m_{j}
$$

and the corresponding action on smooth functions is given by

$$
\begin{aligned}
\left\langle D, \rho_{\tau}(X) \cdot \varphi\right\rangle & =\left\langle\left(-d \tau(X)-\sum_{j, k=1}^{n} x_{j k} \partial_{k} m_{j}\right) \cdot D, \varphi\right\rangle \\
& =\left\langle D,-\tau(X)^{*} \cdot \varphi+\sum_{j, k=1}^{n} \overline{x_{j k}} m_{j} \partial_{k} \cdot \varphi\right\rangle \\
& =\left\langle D,-\tau(X)^{*} \cdot \varphi+d \varphi(\cdot)\left(X^{*} \cdot\right)\right\rangle .
\end{aligned}
$$

We therefore obtain for $X \in \mathfrak{h}$ the formula

$\left(\rho_{\tau}(X) \cdot \varphi\right)(x)=-d \tau(X)^{*} \cdot \varphi(x)+d \varphi(x)\left(X^{*} \cdot x\right)=d \tau(\theta(X)) \cdot \varphi(x)-d \varphi(x)(\theta(X) \cdot x)$. 
From now on we specialize to the case where $F:=F(\lambda)$ and the representation $\tau:=\tau_{\lambda}: \mathfrak{h} \rightarrow \operatorname{End}(F(\lambda))$ is $\tau_{\lambda}:=\pi_{\lambda}^{\mathfrak{k}} \circ c_{\mathfrak{g}}$, where $\left(\pi_{\lambda}^{\mathfrak{k}}, F(\lambda)\right)$ is a unitary highest weight representation of $\mathfrak{k}_{\mathbb{C}}$ and $c_{\mathfrak{g}} \in \operatorname{Aut}\left(\mathfrak{g}_{\mathbb{C}}\right)$ is the Cayley transform (cf. Definition $3.3(\mathrm{~b}))$.

In the following we equip $e^{-\operatorname{tr}} \operatorname{Pol}(V, F(\lambda))$ with the $\mathfrak{g}$-module structure given by the representation $\rho_{\tau_{\lambda}}$ of $\mathfrak{g}$ on $C^{\infty}(V, F(\lambda))$, described in (3.3).

If $\mathfrak{g}$ is a Lie algebra, then we define a filtration of $\mathcal{U}(\mathfrak{g})$ by

$$
\mathcal{U}(\mathfrak{g})^{(n)}=\operatorname{span}_{\mathbb{C}}\left\{X_{1} \cdot \ldots \cdot X_{k}: k \leq n, X_{i} \in \mathfrak{g}\right\}, \quad n \in \mathbb{N}_{0} .
$$

For all $n \in \mathbb{N}_{0}$ we write $\operatorname{Pol}(V)^{(n)}$ for the space of polynomials on $V$ of degree at most $n$.

Lemma 3.8. There exists a unique isomorphism of $\mathfrak{h}$-modules

$$
\Phi: N(\lambda) \rightarrow e^{-\operatorname{tr}} \operatorname{Pol}(V) \otimes F(\lambda)
$$

with $\Phi(v)=e^{-\operatorname{tr}} v$ for $v \in F(\lambda)$. Morover, $\Phi$ satisfies

$$
\Phi\left(\mathcal{U}^{(n)}(\mathfrak{h}) \cdot F(\lambda)\right)=e^{-\operatorname{tr}} \operatorname{Pol}(V)^{(n)} \otimes F(\lambda)
$$

for all $n \in \mathbb{N}_{0}$.

Proof. To prove the existence of $\Phi$ as a homomorphism of $\mathfrak{h}$-modules, in view of the description of $N(\lambda)$ as a module induced from the $(\mathfrak{h} \cap \mathfrak{k}$ )-module $F(\lambda)$ (Lemma 1.6), it suffices to observe that the map

$$
F(\lambda) \rightarrow e^{-\operatorname{tr}} F(\lambda) \subseteq C^{\infty}(V, F), \quad v \mapsto e^{-\operatorname{tr}} . v
$$

is a homomorphism of $\mathfrak{h} \cap \mathfrak{k}$-modules. This is an immediate consequence of the fact that the function $e^{-\operatorname{tr}}$ is invariant under $H \cap K \subseteq \operatorname{Aut}(V)$ because $(\mathfrak{h} \cap \mathfrak{k}) \cdot e=\{0\}$.

Next we show that $\Phi$ is injective. Let $v_{1}, \ldots, v_{m}$ denote a basis of $V$, so that the Jordan left multiplications $L\left(v_{j}\right)$ form a basis of $\mathfrak{h} \cap \mathfrak{p}$. Also, write $\mathcal{U}(\mathfrak{h} \cap \mathfrak{p})$ for the subspace of $\mathcal{U}(\mathfrak{h})$ spanned by the ordered products of the form $L\left(v_{1}\right)^{k_{1}} \cdots L\left(v_{m}\right)^{k_{m}}$. We will use the vector space isomorphism

$$
N(\lambda) \cong \mathcal{U}(\mathfrak{h}) \otimes_{\mathcal{U}(\mathfrak{h} \cap \mathfrak{k})} F(\lambda) \cong \mathcal{U}(\mathfrak{h} \cap \mathfrak{p}) \otimes F(\lambda) .
$$

For $X \in \mathfrak{h}$ and $p \in \operatorname{Pol}(V) \otimes F(\lambda)$ we obtain from Lemma 2.7(1) for all $v \in V$ the formula

$$
\begin{aligned}
& d \pi_{\lambda}(X) \cdot\left(e^{-\operatorname{tr}} p\right)(y) \\
= & d \tau_{\lambda}(\theta(X)) \cdot e^{-\operatorname{tr}(y)} p(y)-(y, X . e) e^{-\operatorname{tr}(y)} p(y)-e^{-\operatorname{tr}(y)} d p(y) \cdot(\theta(X) \cdot y) .
\end{aligned}
$$

Inductively this leads for $u=L\left(x_{1}\right) \cdot \ldots \cdot L\left(x_{n}\right), x_{j} \in V$, to

$$
\Phi(u \otimes v)(y)=e^{-\operatorname{tr}(y)}\left(\left(\prod_{j=1}^{n}\left(y, x_{j}\right)\right) v+\text { polynomials of order }<n\right) .
$$

From (3.5) we deduce that $\Phi$ is injective.

We also conclude inductively with (3.5) that

$$
\Phi\left(\mathcal{U}^{(n)}(\mathfrak{h}) \otimes F(\lambda)\right)=e^{-\operatorname{tr}} \operatorname{Pol}(V)^{(n)} \otimes F(\lambda)
$$

for all $n \in \mathbb{N}_{0}$, and this implies in particular that $\Phi$ is surjective. 
Remark 3.9. There are several actions of the group $H$ which will be used in the following:

(a) First we recall the action on $\operatorname{Hol}\left(T_{\Omega}, F(\lambda)\right)$ given by

$$
\left(\pi_{\tau}(h) . f\right)(z)=\tau_{\lambda}(h) \cdot f\left(h^{-1} \cdot z\right),
$$

and on the space $C^{\infty}(V, F(\lambda))$ we consider the action given by

$$
(h . \varphi)(x)=\tau_{\lambda}(\theta(h)) . f\left(\theta(h)^{-1} \cdot x\right)
$$

which is the integrated form of the representation $\rho_{\tau}$ of $\mathfrak{h}$ (Lemma 3.7). On the space $C^{\infty}(V)$ we consider the action given by $(h . \varphi)(x):=\varphi\left(h^{-1} . x\right)$. Via $(h . D)(\varphi):=$ $D\left(h^{-1} . \varphi\right)$ we further obtain an action on $\mathcal{D}^{\prime}(V, F(\lambda))$ which is compatible with the action of $\mathfrak{h}$ and given on $D \otimes v$ by

$$
\begin{aligned}
\langle h .(D \otimes v), \varphi \otimes w\rangle & =\left\langle D \otimes v, h^{-1} \cdot(\varphi \otimes w)\right\rangle=\left\langle D \otimes v, \theta(h)^{-1} \cdot \varphi \otimes \tau_{\lambda}(h)^{*} . w\right\rangle \\
& =\left\langle\theta(h) . D \otimes \tau_{\lambda}(h) \cdot v, \varphi \otimes w\right\rangle,
\end{aligned}
$$

i.e.,

$$
h .(D \otimes w)=\theta(h) . D \otimes \tau_{\lambda}(h) . v .
$$

This action preserves the subspace $\mathrm{SO}(F(\lambda))$, and the Fourier transform

$$
\mathcal{F}: \mathrm{SO}(F(\lambda)) \rightarrow \operatorname{Hol}\left(T_{\Omega}, F(\lambda)\right)
$$

intertwines the $H$-actions on both spaces. On the level of the derived representation this is satisfied by definition, and on the group level it either follows from the connectedness of $H$ and the smoothness of the actions, or directly by calculation.

(b) Further we consider on the space $\operatorname{End}(F(\lambda))$ the $H$-action given by $h . A:=$ $\tau_{\lambda}(h) A \tau_{\lambda}(h)^{*}$, and the induced action on $\mathrm{SO}(\operatorname{End}(F(\lambda))$ given by

$$
h .(D \otimes A)=\theta(h) . D \otimes h . A .
$$

(c) We also have a natural map

$$
\mathrm{SO}(\operatorname{End}(F(\lambda))) \times C^{\infty}(V, F(\lambda)) \rightarrow \mathcal{D}^{\prime}(V, F(\lambda)), \quad(D \otimes A) \cdot(\varphi \otimes v) \mapsto \varphi D \otimes A . v .
$$

The observation that $h .(\varphi D)=(h . \varphi)(h . D)$ holds for the multiplication of distributions with smooth functions, together with (a) and (b), shows that this map is $H$-equivariant.

Theorem 3.10. There exists a unique tempered distribution $R_{\lambda} \in \mathrm{SO}(\operatorname{End}(F(\lambda))$ with

$$
\mathcal{F}\left(R_{\lambda}\right)(z)=\tau_{\lambda}(\widetilde{P}(-i z)) \quad \text { for all } \quad z \in T_{\Omega} .
$$

This distribution is $H$-invariant.

Proof. The existence of $R_{\lambda}$ follows from [17, Th. V.8]. The formula $P(h . x)=$ $h P(x) h^{\top}$ for $h \in \operatorname{Str}(V)$ and $x \in V$ implies that

$$
\mathcal{F}\left(R_{\lambda}\right)(z)=\tau_{\lambda}(h) \mathcal{F}\left(R_{\lambda}\right)\left(h^{-1} . z\right) \tau_{\lambda}(h)^{*} \quad \text { for } \quad z \in T_{\Omega}, h \in H .
$$

It is easy to see that the action of $H$ on $\operatorname{Hol}\left(T_{\Omega}, \operatorname{End}(F(\lambda))\right)$ given by the formula on the right-hand side is intertwined by the Fourier transform with the action on $\mathrm{SO}(\operatorname{End}(F(\lambda)))$, so that the injectivity of the Fourier transform implies that $R_{\lambda}$ is fixed by $H$. 
Theorem 3.11 (Equivariance Theorem). The map

$$
\Psi: N(\lambda) \rightarrow \mathrm{SO}(F(\lambda)), \quad v \mapsto R_{\lambda} . \Phi(v)
$$

is $\mathfrak{g}$-equivariant, and its range $R_{\lambda} \cdot e^{-\operatorname{tr}} \operatorname{Pol}(V, F(\lambda))$ is isomorphic to $L(\lambda)$.

Proof. Since the Fourier transform $\mathcal{F}: \mathrm{SO}(F(\lambda)) \rightarrow \operatorname{Hol}\left(T_{\Omega}, F(\lambda)\right)$ is by definition equivariant with respect to the action of $\mathfrak{g}$ on both sides, it suffices to show that the composition

$$
\mathcal{F} \circ \Psi: N(\lambda) \rightarrow \operatorname{Hol}\left(T_{\Omega}, F(\lambda)\right)
$$

is equivariant. The operator

$$
\begin{gathered}
C_{\lambda}: \operatorname{Hol}(\mathcal{D}, F(\lambda)) \rightarrow \operatorname{Hol}\left(T_{\Omega}, F(\lambda)\right), \\
\left(C_{\lambda} \cdot f\right)(z):=\tau_{\lambda}\left(\widetilde{P}\left(\frac{z+i e}{2 i}\right)\right) \cdot f\left((z-i e)(z+i e)^{-1}\right)
\end{gathered}
$$

intertwines the natural representations of the covering group of $\operatorname{Aut}(\mathcal{D})_{0}$, resp. $G$, on both spaces (cf. [11, Lemma X.4.4]). The subspace $F(\lambda)$, as a $\widetilde{\mathfrak{k}}$-module, is embedded into $\operatorname{Hol}(\mathcal{D}, F(\lambda))$ as the subspace of the constant functions, the highest $\widetilde{\mathfrak{k}}$-type, and this subspace generates a simple highest weight module of $\widetilde{\mathfrak{g}}$ of highest weight $\lambda$ (cf. [26, Prop. XII.2.2]). Let $d_{\lambda}>0$ be the number determined by $\tau_{\lambda}\left(\frac{1}{4} \mathbf{1}\right)=d_{\lambda} \mathbf{1}$. Then we obtain for $v \in F(\lambda) \subseteq \operatorname{Hol}(\mathcal{D}, F(\lambda))$ the formula

$$
\begin{aligned}
C_{\lambda}(v)(z) & =\tau_{\lambda}\left(\widetilde{P}\left(\frac{z+i e}{2 i}\right)\right) \cdot v=\tau_{\lambda}\left(\frac{1}{4} \mathbf{1}\right) \tau_{\lambda}\left(\widetilde{P}\left(\frac{z+i e}{i}\right)\right) \cdot v \\
& =d_{\lambda} \mathcal{F}\left(R_{\lambda}\right)(z+i e) \cdot v=d_{\lambda} \mathcal{F}\left(R_{\lambda} \cdot v\right)(z+i e)=d_{\lambda} \mathcal{F}\left(R_{\lambda} \cdot e^{-\operatorname{tr}} v\right)(z) .
\end{aligned}
$$

This implies that the highest $K$-type for the $G$-representation in $\operatorname{Hol}\left(T_{\Omega}, F(\lambda)\right)$ is given by the subspace $\mathcal{F} \circ \Psi(F(\lambda))$ which is annihilated by $\mathfrak{p}^{+}$.

Now the definition of $N(\lambda)$ as a $\mathfrak{g}$-module induced from the $\mathfrak{p}^{+} \rtimes \mathfrak{k}_{\mathbb{C}}$-module $F(\lambda)$ implies the existence of a $\mathfrak{g}$-equivariant map $\Psi_{1}: N(\lambda) \rightarrow \operatorname{Hol}\left(T_{\Omega}, F(\lambda)\right)$. Its range is the $\mathfrak{g}$-module generated by $\mathcal{F} \circ \Psi(F(\lambda))$ which is a simple highest weight module of highest weight $\lambda$, hence isomorphic to $L(\lambda)$.

Since $R_{\lambda}$ is $H$-invariant (Theorem 3.10), the multiplication map

$$
e^{-\operatorname{tr}} \operatorname{Pol}(V, F(\lambda)) \rightarrow \mathrm{SO}(F(\lambda)), \quad \varphi \mapsto R_{\lambda} \cdot \varphi
$$

is $H$-equivariant (cf. Remark 3.9(c)). Now Lemma 3.8 implies that $\Psi$ is $\mathfrak{h}$-equivariant. In view of Proposition 1.5, this shows that $\mathcal{F} \circ \Psi$ maps $N(\lambda)=\mathcal{U}(\mathfrak{h}) . F(\lambda)$ surjectively onto $\mathcal{U}(\mathfrak{h}) .(\mathcal{F} \circ \Psi)(F(\lambda)) \cong L(\lambda)$. Therefore $\mathcal{F} \circ \Psi$ and $\Psi_{1}$ can be viewed as two $\mathfrak{h}$-equivariant linear maps $N(\lambda) \rightarrow F(\lambda)$ which coincide on $F(\lambda)$. Now $\operatorname{ker}\left(\mathcal{F} \circ \Psi-\Psi_{1}\right)$ is an $\mathfrak{h}$-invariant subspace of $N(\lambda)$ containing $F(\lambda)$, hence coincides with $N(\lambda)$ (Proposition 1.5). This proves that $\mathcal{F} \circ \Psi=\Phi_{1}$ is $\mathfrak{g}$-equivariant.

The Equivariance Theorem has several interesting consequences.

Corollary 3.12. 1 . The subspace $R_{\lambda} \cdot e^{-\operatorname{tr}} \mathrm{Pol}(V, F(\lambda))$ is a $\mathfrak{g}$-submodule of $\mathrm{SO}(F(\lambda))$ isomorphic to $L(\lambda)$.

2. $\operatorname{ker} \Psi=J(\lambda)$ is the maximal submodule of $N(\lambda)$.

3. $J(\lambda)=\left\{\varphi \in N(\lambda) \cong e^{-\operatorname{tr}} \operatorname{Pol}(V, F(\lambda)): R_{\lambda} \cdot \varphi=0\right\}$.

Lemma 3.13. Let $f=\sum_{j=0}^{n} f_{j} \in J(\lambda)$ with $f_{j}=e^{-\operatorname{tr}} p_{j}$ and $p_{j} \in \operatorname{Pol}(V, F(\lambda))$ homogeneous of degree $j$. Then we have $f_{j} \in J(\lambda)$ for all $0 \leq j \leq n$. 
Proof. Recall that $L(e)$ is the generator of $\mathfrak{z}(\mathfrak{h}) \subseteq \mathfrak{h} \cap \mathfrak{p}$. From (3.4) we obtain for all $x \in \operatorname{supp} R_{\lambda}$ :

$$
d \rho_{\lambda}(L(e) . f)(x)=-d \tau_{\lambda}(L(e)) . f(x)-(x, e) f(x)+\sum_{j=0}^{n} j f_{j}(x) .
$$

Clearly we have $d \tau_{\lambda}(L(e)) . f(x) \in \mathbb{C} f(x) \subseteq J(\lambda)$, and by Corollary 3.12.3 we also have tr. $f \in J(\lambda)$, so that $\sum_{j=0}^{n} j f_{j} \in J(\lambda)$. This means that $J(\lambda)$ is invariant under the operator $E(f):=\sum_{j=0}^{n} j f_{j}$, hence adapted to the eigenspace decomposition of E.

\section{Unitary highest Weight REPRESEntations For CAYley type SPACES}

In this section we now turn to unitary highest weight representations in the setup studied in Section 2. We will recall the realization of unitary highest weight representations in spaces of vector valued square integrable functions on a homogeneous cone. Then we give a new and quite explicit characterization of the $K$-finite vectors in the cone realization which will easily follow from the more general results in Section 2.

We keep the notation from Section 3. In particular $G$ is the simply connected covering group of $\operatorname{Aut}\left(T_{\Omega}\right)_{0}$ and $K$ and $H$ are connected subgroups of $G$.

Let $\left(\pi_{\lambda}, \mathcal{H}_{\lambda}\right)$ be a unitary highest weight representation of $G$ with highest weight $\lambda \in i \mathrm{t}^{*}$ and with respect to $\widehat{\Delta}_{k}^{+}$. We write $\left(\pi_{\lambda}^{K}, F(\lambda)\right)$ for the corresponding irre-

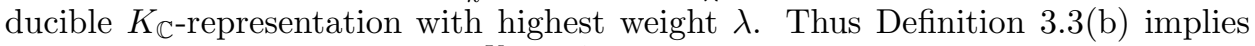
that the prescription $d \tau_{\lambda}:=d \pi_{\lambda}^{K} \circ c_{\mathfrak{g}}^{-1}$ integrates to a holomorphic representation $\left(\tau_{\lambda}, F(\lambda)\right)$ of $H_{\mathbb{C}}$.

Theorem 4.1. Let $R_{\lambda} \in \mathrm{SO}(\operatorname{End}(F(\lambda))$ denote the distribution with

$$
\mathcal{L}\left(R_{\lambda}\right)(z)=\tau_{\lambda}(\widetilde{P}(-i z)), \quad z \in T_{\Omega} .
$$

Then the highest weight module $L(\lambda)$ of $\mathfrak{g}$ is unitarizable if and only if $R_{\lambda}$ is a measure with values in the cone $\operatorname{Herm}^{+}(F(\lambda))$ of positive operators on $F(\lambda)$. If this is the case, then the following assertions hold:

1. Let

$$
L^{2}\left(\bar{\Omega}, R_{\lambda}\right):=\left\{f: \bar{\Omega} \rightarrow F(\lambda) \text { meas. : } \int_{\bar{\Omega}}\left\langle d R_{\lambda}(x) . f(x), f(x)\right\rangle<\infty\right\} .
$$

Then the Fourier transform

$$
\begin{gathered}
\mathcal{F}_{\lambda}: L^{2}\left(\bar{\Omega}, R_{\lambda}\right) \rightarrow \mathcal{H}_{\lambda} \subseteq \operatorname{Hol}\left(T_{\Omega}, F(\lambda)\right), \\
\mathcal{F}_{\lambda}(f)(w):=\int_{\bar{\Omega}} e^{i(w, x)} d R_{\lambda}(x) . f(x)
\end{gathered}
$$

yields an isomorphism on the reproducing kernel Hilbert space $\mathcal{H}_{\lambda}$ with kernel

$$
\tau_{\lambda}\left(\left(\widetilde{P}\left(\frac{z-\bar{w}}{2 i}\right)\right) .\right.
$$

2. If we realize the unitary highest weight representation $\left(\pi_{\lambda}, \mathcal{H}_{\lambda}\right)$ of $G$ in the space $L^{2}\left(\bar{\Omega}, R_{\lambda}\right)$ as in 1 , then the action of the parabolic subgroup $V \rtimes H$ is given by

$$
\left(\pi_{\lambda}(v, h) . f\right)(x)=e^{-i(v, x)} \tau_{\lambda}(\theta(h)) . f\left(\theta(h)^{-1} \cdot x\right) .
$$


Proof. For the first statement we have to combine several results. First [26, Th. XII.2.6] says that $L(\lambda)$ is unitarizable if the natural $\operatorname{End}(F(\lambda))$-valued kernel on $\mathcal{D}$ is positive definite, which by the Cayley transform is equivalent to the positive definiteness of the function $\tau_{\lambda} \circ \widetilde{P}$ on $T_{\Omega}$. This in turn is equivalent to $R_{\lambda}$ being a $\operatorname{Herm}^{+}(F(\lambda))$-valued measure ([17, Th. V.12], see also [5, Th. 3.3]).

1. 5. p. 233]

2. This is immediate from [5] Th. 3.4].

The preceding result is the appropriate version of Clerc's results that we need in our context. For the scalar case $(\operatorname{dim} F(\lambda)=1)$, similar results were obtained by Vergne and Rossi in [32].

Remark 4.2. Suppose that $R_{\lambda}$ is a $\operatorname{Herm}^{+}(F(\lambda))$-valued measure. Then for each element $\varphi \in L^{2}\left(\bar{\Omega}, R_{\lambda}\right)$ we can view $R_{\lambda} \cdot \varphi$ as an element of $\mathrm{SO}(F(\lambda))$ and obtain

$$
\mathcal{F}\left(R_{\lambda} \cdot \varphi\right)=\mathcal{F}_{\lambda}(\varphi)
$$

Since the maps $\mathcal{F}$ and $\mathcal{F}_{\lambda}$ are $\mathfrak{g}$-equivariant, it follows from Lemma 3.7 and Theorem 3.11 that the action of $\mathfrak{g}$ on the subspace $L^{2}\left(\bar{\Omega}, R_{\lambda}\right)^{\infty}$ of smooth vectors is given by the natural action on $\mathrm{SO}(F(\lambda))$. For the subalgebra $\mathfrak{n}^{+} \rtimes \mathfrak{h}$ it is given by the formulas in Lemma 3.7.

In the following lemma the assumption that $L(\lambda)$ is unitarizable is used to ensure that $J(\lambda)$ is a cyclic module.

Lemma 4.3. Suppose that $N(\lambda)$ is unitarizable and of reduction level $k$. Then there exists an element $v_{k} \in \mathcal{U}(\mathfrak{h})^{(k)} \cdot v_{\lambda} \backslash \mathcal{U}(\mathfrak{h})^{(k-1)} \cdot v_{\lambda}$ such that the maximal submodule $J(\lambda) \subseteq N(\lambda)$ is given by $J(\lambda)=\mathcal{U}(\mathfrak{h}) \cdot v_{k}$.

Proof. In view of Lemma 3.8, we may identify in the following the $\mathfrak{h}$-module $N(\lambda)$ with the space $e^{-\operatorname{tr}} \operatorname{Pol}(V) \otimes F(\lambda)$. Comparing dimensions in the following chain of inclusions

$$
\mathcal{U}\left(\mathfrak{p}^{-}\right)^{(n)} \otimes F(\lambda)=\mathcal{U}(\mathfrak{g})^{(n)} \cdot F(\lambda) \supseteq \mathcal{U}(\mathfrak{h})^{(n)} \cdot F(\lambda)=e^{-\operatorname{tr}} \operatorname{Pol}(V)^{(n)} \otimes F(\lambda)
$$

yields equality, so that the assertion follows from Proposition 1.5 and Theorem 2.4 .

Identification on central half lines. Let $\lambda^{0}$ be the highest weight of $\pi_{\lambda^{0}}^{\mathfrak{k}}$ with respect to the Cartan subalgebra $t$. Then

$$
\left.\lambda^{0} \circ c_{\mathfrak{g}}\right|_{\mathfrak{b}}=\sum_{j=1}^{r} \frac{m_{j}}{2} \varepsilon_{j} \quad \text { with } \quad m_{j}-m_{i} \in \mathbb{N}_{0}, \quad i<j
$$

(Definition 3.3(b)). We assume, in addition, that $m_{r}=0$. Then $m_{j} \in-\mathbb{N}_{0}$ for all $j$. For $u \in \mathbb{R}$ we then set

$$
\lambda:=\lambda^{u}:=\lambda^{0}-u \zeta,
$$

where $\zeta \in[\mathfrak{k}, \mathfrak{k}]^{\perp}$ is determined by $\left.\zeta \circ c_{\mathfrak{g}}\right|_{\mathfrak{b}}=\frac{1}{2} \sum_{j} \varepsilon_{j}$ which is equivalent to $\zeta(\check{\gamma})=1$ for $\gamma \in \widehat{\Delta}_{n}^{+}$long because for the highest root $\gamma \in \widehat{\Delta}_{n}^{+}$the corresponding restricted root is $\varepsilon_{r}$ with the coroots $2 L\left(c_{r}\right)$ (cf. [24, Sect. IV]). Then $\lambda$ is the highest weight of a finite-dimensional irreducible representation $\left(\tau_{\lambda}, F(\lambda)\right)$ of $H$ which can be written as

$$
\tau_{\lambda}=\tau_{\lambda^{0}} \otimes \tau_{-\frac{u}{2} \varepsilon},
$$


where $\tau_{-\frac{u}{2} \varepsilon}$ is a one-dimensional representation. Accordingly, we may identify $F\left(\lambda^{u}\right)$ for all $u \in \mathbb{C}$ with the fixed vector space $F_{0}:=F\left(\lambda^{0}\right)$.

Recall the definition of the Jordan algebra determinant $\Delta:=\operatorname{det}$ from [11, p. $29]$. For each $u \in \mathbb{R}$ we denote by $R_{u}$ the Riesz distribution with parameter $u \in \mathbb{C}$ on $\bar{\Omega}$ which are uniquely determined by

$$
\mathcal{F}\left(R_{u}\right)(z)=\Delta(-i z)^{-u}, \quad z \in T_{\Omega}, u \in \mathbb{C}
$$

(cf. [11, Sect. VII.2]). By a theorem of Gindikin (cf. [11, Th. VII.3.2]), $R_{u}$ is a positive measure if and only if

$$
\left.u \in\left\{0, \frac{d}{2}, \ldots(r-1) \frac{d}{2}\right\} \cup\right](r-1) \frac{d}{2}, \infty[
$$

where $d:=\operatorname{dim} \mathfrak{g}^{\frac{1}{2}\left(\varepsilon_{i}+\varepsilon_{j}\right)}, i \neq j$, is obtained from the restricted root decomposition of $\mathfrak{g}$ with respect to $\mathfrak{b}$. Moreover we have $\operatorname{supp}\left(R_{u}\right)=\bar{\Omega}$ with $R_{u}(\bar{\Omega} \backslash \Omega)=0$ for $u>(r-1) \frac{d}{2}$ and $\operatorname{supp}\left(R_{u}\right)=\overline{\mathcal{O}_{k}}$ with $R_{u}\left(\overline{\mathcal{O}_{k}} \backslash \mathcal{O}_{k}\right)=0$ for $u=k \frac{d}{2}, 0 \leq k \leq r-1$.

By a more detailed study of the vector valued measure $R_{\lambda}$, one can retrieve useful information on $R_{\lambda}$ which we collect in the next theorem.

Theorem 4.4. If $R_{\lambda^{u}}$ is a $\operatorname{Herm}^{+}\left(F\left(\lambda^{u}\right)\right)$-valued measure, then:

1. There exists a polynomial map $D_{u}: V \rightarrow \operatorname{Herm}^{+}\left(F\left(\lambda^{u}\right)\right)$ with $R_{\lambda}=D_{u} R_{u}$.

2. $\left.u \in\left\{0, \frac{d}{2}, \ldots,(r-1) \frac{d}{2}\right\} \cup\right](r-1) \frac{d}{2}, \infty[$.

3. If $u>(r-1) \frac{d}{2}$, then

$$
\operatorname{supp}\left(R_{\lambda^{u}}\right)=\operatorname{supp}\left(R_{u}\right)=\bar{\Omega} .
$$

4. For $u=k \frac{d}{2}, k \in\{0, \ldots, r-1\}$ one has

$$
\operatorname{supp}\left(R_{\lambda^{u}}\right)=\operatorname{supp}\left(R_{u}\right)=\overline{\mathcal{O}_{k}} .
$$

Proof. [17 Th. V.17]

The space of $K$-finite vectors. We now determine explicitly the space of $K$-finite vectors for a unitary highest weight representation of $G$ realized in $L^{2}\left(\bar{\Omega}, R_{\lambda}\right)$. Since the $K$-action in the cone realization is rather nasty (cf. [7]), there is no obvious description of this space. Our approach is based on the action of the maximal parabolic subalgebra $\mathfrak{h} \ltimes \mathfrak{n}^{+}$which already led in Section 2 to the right subspace of $\mathrm{SO}(F(\lambda))$.

Recall the decomposition of the $\operatorname{ring} \operatorname{Pol}(V)$ of polynomials into irreducible $H$ modules (cf. [10 Th. 2.1], [11, Th. XI.2.4]): The $H$-decomposition is muliplicity free and given by

$$
\operatorname{Pol}(V)=\bigoplus_{\mathbf{m} \geq 0} \operatorname{Pol}(V)_{\mathbf{m}}
$$

where $\mathbf{m}=\left(m_{1}, \ldots, m_{r}\right)$ and the condition $\mathbf{m} \geq 0$ means that $m_{1} \geq m_{2} \geq \ldots \geq$ $m_{r} \geq 0$ and $m_{i} \in \mathbb{Z}$. The space $\operatorname{Pol}(V)_{\mathbf{m}}$ is a real highest weight module of $H$ with highest weight $\lambda_{\mathbf{m}}=\sum_{j=1}^{r} m_{j} \varepsilon_{j} \in \mathfrak{b}^{*}$ w.r.t. $-\Sigma^{+}$which is generated by the highest weight vector $\Delta_{\mathbf{m}}$ (for the definition of the generalized power functions $\Delta_{\mathbf{m}}$ we refer to [11, p. 122]).

Recall that the Jordan algebra trace $\operatorname{tr}$ on $V$ satisfies $\operatorname{tr}(x)=(x, e)$ for all $x \in V$.

Theorem 4.5 (Description of $K$-finite vectors). Suppose that $\left(\pi_{\lambda}, \mathcal{H}_{\lambda}\right)$ is a unitary highest weight representation of the simply connected hermitian group $G$ of tube type realized as $L^{2}\left(\bar{\Omega}, R_{\lambda}\right)$. Then the space $L^{2}\left(\bar{\Omega}, R_{\lambda}\right)^{K}$ of $K$-finite vectors of 
$\left(\pi_{\lambda}, \mathcal{H}_{\lambda}\right)$ is the image of the space $e^{-\operatorname{tr}} \operatorname{Pol}(V, F(\lambda))$ in $L^{2}\left(\bar{\Omega}, R_{\lambda}\right)$, and a highest weight vector of $\left(\pi_{\lambda}, \mathcal{H}_{\lambda}\right)$ is given by the function $e^{-\mathrm{tr}} \cdot v_{\lambda}$, where $v_{\lambda}$ is a highest weight vector of $\left(\tau_{\lambda}, F(\lambda)\right)$.

Proof. We identify $L^{2}\left(\bar{\Omega}, R_{\lambda}\right)$ with the subspace

$$
R_{\lambda} \cdot L^{2}\left(\bar{\Omega}, R_{\lambda}\right) \subseteq \mathrm{SO}(F(\lambda))
$$

which is compatible with the $\mathfrak{g}$-action on the space of smooth vectors, because the Fourier transform

$$
\mathcal{F}: R_{\lambda} \cdot L^{2}\left(\bar{\Omega}, R_{\lambda}\right)^{\infty} \rightarrow \operatorname{Hol}\left(T_{\Omega}, F(\lambda)\right)
$$

is $\mathfrak{g}$-equivariant (cf. Remark 4.2).

Since $R_{\lambda}$ is a tempered distribution, all restrictions of the functions in the space $e^{-\operatorname{tr}} \operatorname{Pol}(V, F(\lambda))$ are contained in $L^{2}\left(\bar{\Omega}, R_{\lambda}\right)$. In view of Theorem 3.11, the subspace $R_{\lambda} \cdot e^{-\operatorname{tr}} \operatorname{Pol}(V, F(\lambda))$ is a $\mathfrak{g}$-module isomorphic to $L(\lambda)$, hence contained in $L^{2}\left(\bar{\Omega}, R_{\lambda}\right)^{\infty}$, and therefore in $L^{2}\left(\bar{\Omega}, R_{\lambda}\right)^{K}$. On the other hand, the fact that the representation of $G$ on $L^{2}\left(\bar{\Omega}, R_{\lambda}\right)$ is a unitary highest weight representation implies that $L^{2}\left(\bar{\Omega}, R_{\lambda}\right)^{K}$ is a simple $\mathfrak{g}$-module, which yields equality. The description of the highest weight vector follows from Theorem 3.11.

In the scalar case Theorem 4.5 has been proved by completely different methods by J. Faraut and A. Korányi (cf. [11, Prop. XIII.3.2]).

For every $0 \leq k \leq r-1$ we write

$$
I_{k}:=\bigoplus_{\mathbf{m} \geq 0, m_{k+1}>0} \operatorname{Pol}(V)_{\mathbf{m}}=\left\{f \in \operatorname{Pol}(V):\left.f\right|_{\mathcal{O}_{k}}=0\right\}
$$

for the ideal defined by $\mathcal{O}_{k}$. For the asserted equality, we note that the ideal defined by $\mathcal{O}_{k}$ is $H$-invariant, hence adapted to the $H$-decomposition (4.2) of $\operatorname{Pol}(V)$. Therefore one only has to note that for $\mathbf{m} \geq 0$ the function $\Delta_{\mathbf{m}}$ vanishes on $\mathcal{O}_{k}$ if and only if $m_{k+1}>0$ (cf. [17, Prop. I.4]).

The following proposition provides a description of the maximal submodule $J(\lambda) \subseteq N(\lambda)$ in the picture of Theorem 4.5.

Proposition 4.6. Suppose that $L(\lambda)$ is unitarizable and write $R_{\lambda}=D_{u} R_{u}$ with the polynomial density function $D_{u}$. Identifying $N(\lambda)$ with $e^{-\operatorname{tr}} \operatorname{Pol}(V, F(\lambda))$, we have

$$
J(\lambda)=\left\{e^{-\operatorname{tr}} f \in N(\lambda): D_{u} \cdot f=0 \text { on } \operatorname{supp}\left(R_{u}\right)\right\} .
$$

For $\lambda=\lambda^{u}, u=k \frac{d}{2}, k \in\{0, \ldots, r-1\}$, we obtain in particular $J(\lambda) \supseteq e^{-\operatorname{tr}} I_{k} \otimes$ $F(\lambda)$.

Proof. In view of Theorem 4.5, the natural $\mathfrak{g}$-contravariant hermitian form on

$$
N(\lambda) \cong e^{-\operatorname{tr}} \operatorname{Pol}(V, F(\lambda))
$$

is given by

$$
\begin{aligned}
\left\langle e^{-\operatorname{tr}} f, e^{-\operatorname{tr}} g\right\rangle & =\int_{\bar{\Omega}} e^{-2 \operatorname{tr}(x)}\left\langle d R_{\lambda}(x) \cdot f(x), g(x)\right\rangle \\
& =\int_{\bar{\Omega}} e^{-2 \operatorname{tr}(x)}\left\langle D_{u}(x) . f(x), g(x)\right\rangle d R_{u}(x) .
\end{aligned}
$$

This implies the assertion because $J(\lambda)=\left\{e^{-\operatorname{tr}} f \in N(\lambda):\left\langle e^{-\operatorname{tr}} f, e^{-\operatorname{tr}} f\right\rangle=0\right\}$. 
For the special case described in the second part of the statement, we only have to observe that $\operatorname{supp}\left(R_{\lambda}\right)=\overline{\mathcal{O}_{k}}$ which implies that $R_{\lambda} \cdot e^{-\operatorname{tr}} I_{k} \otimes F(\lambda)=\{0\}$ in this case. This completes the proof.

\section{The Classification for CAYley type SPACES}

In this section we give an explicit description of the $H$-fixed distribution vector of a spherical unitary highest weight representation $\left(\pi_{\lambda}, \mathcal{H}_{\lambda}\right)$ of a simply connected hermitian group $G$ of tube type (cf. Theorem 5.1). In the case where $\operatorname{supp}\left(R_{\lambda}\right)$ is smaller than $\bar{\Omega}$ our approach yields a complete classification of all spherical unitary highest weight representations of $G$.

The $H$-invariant distributions. In this section we assume that $\left(\tau_{\lambda}, F(\lambda)\right)$ is a $H \cap K$-spherical representation of $H$. In view of the Appendix A in [17, this means that it can be viewed as a real highest weight representation with respect to the restricted root decomposition of $\mathfrak{h}$ with respect to $\mathfrak{b}$. We write

$$
\lambda=\lambda^{s}:=\sum_{j=1}^{r} \frac{m_{j}-s}{2} \varepsilon_{j}
$$

with $m_{1} \leq \ldots \leq m_{k}<m_{k+1}=\ldots=m_{r}=0$ and $s \in \mathbb{R}$. Then the corresponding

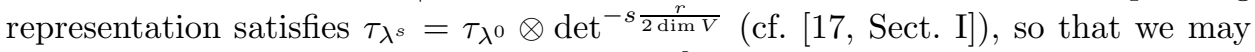
identify all the spaces $F\left(\lambda^{s}\right)$ with $F_{0}:=F\left(\lambda^{0}\right)$.

According to [25, Th. II.11], there exists for each $v_{0} \in F\left(\lambda^{s}\right)^{\mathfrak{h} \cap \mathfrak{k}} \cong F_{0}^{\mathfrak{h} \cap \mathfrak{k}}$ a unique $H$-invariant holomorphic function

$$
f_{s}: T_{\Omega} \rightarrow F\left(\lambda^{s}\right) \quad \text { with } \quad f_{s}(i e)=v_{0} .
$$

Note that the $H$-invariance of $f$ with respect to the action $(h . f)(z)=\tau_{\lambda^{s}}(h) \cdot f\left(h^{-1} . z\right)$ means that $f$ is an $H$-equivariant map. The uniqueness of $f$ is a direct consequence of the fact that $f$ is uniquely determined by its restriction to $i \Omega=H . i e \subseteq T_{\Omega}$.

Theorem 5.1. There exists a weakly holomorphic map $\mathbb{C} \rightarrow \mathcal{S}^{\prime}\left(V, F\left(\lambda^{0}\right)\right), s \mapsto$ $R_{\lambda^{s}}^{H}$ with $\left.\mathcal{F}\left(R_{\lambda^{s}}^{H}\right)\right|_{T_{\Omega}}=f_{s}$, and $R_{\lambda^{s}}^{H}$ is supported by $\bar{\Omega}$. Suppose that

$$
m_{1} \leq \ldots \leq m_{k}<m_{k+1}=\ldots=m_{r}=0 .
$$

Then the tempered distribution $R_{\lambda^{s}}^{H}$ is a measure if and only if

$$
\left.\frac{s}{2} \in\left\{k \frac{d}{2}, \ldots,(r-1) \frac{d}{2}\right\} \cup\right](r-1) \frac{d}{2}, \infty[.
$$

If this is the case, then

$$
R_{\lambda^{s}}^{H}=\left(\frac{s}{2}\right)_{-\mathbf{m}}^{-1} \eta \cdot R_{\frac{s}{2}}
$$

where

$$
\left(\frac{s}{2}\right)_{-\mathbf{m}}=\prod_{j=1}^{r} \prod_{l=0}^{-m_{j}-1}\left(\frac{s}{2}-(j-1) \frac{d}{2}+l\right)=\prod_{j=1}^{k} \prod_{l=0}^{-m_{j}-1}\left(\frac{s}{2}-(j-1) \frac{d}{2}+l\right),
$$

$\eta: V \rightarrow F\left(\lambda^{0}\right)$ is a polynomial map with $\eta(g . e)=\tau_{\lambda^{0}}(\theta(g)) . v_{0}$ for $g \in H$, and $R_{s}$, $s \in \mathbb{C}$, are the Riesz measures on $\bar{\Omega}$ with $\mathcal{F}\left(R_{s}\right)(z)=\Delta(-i z)^{-s}$.

Proof. The existence of the weakly holomorphic family $R_{\lambda^{s}}^{H}, s \in \mathbb{C}$, follows from 17 . Prop. V.2]. The characterization of those parameters for which $R_{\lambda^{s}}^{H}$ is a measure is a consequence of [17, Props. III.8 and 5.5]. That the density $\eta$ is a polynomial follows from [17] Props. II.8]. 
Remark 5.2. (a) The polynomial $\left(\frac{s}{2}\right)_{-\mathbf{m}}$ vanishes in the points of the form $(j-1) \frac{d}{2}-$ $l$, where $1 \leq j \leq k$ and $0 \leq l \leq-m_{j}-1$. Therefore the maximal zero is $s=(k-1) d$, which is the place where the distribution $R_{\lambda^{s}}^{H}$ no longer is a measure. The values $s=j d$ correspond to measures on the boundary components $\mathcal{O}_{j}, k \leq j \leq r-1$. For $j<k$ the density function $\eta$ vanishes on $\mathcal{O}_{j}$ ([17 Prop. II.12]), which somehow cancels with the zero of $\left(\frac{s}{2}\right)_{-\mathbf{m}}$, but the resulting distribution is not a measure.

(b) Since the Fourier transform $\mathcal{F}: \mathcal{S}_{\bar{\Omega}}^{\prime}(V, F(\lambda)) \rightarrow \operatorname{Hol}\left(T_{\Omega}, F(\lambda)\right)$ is injective and equivariant with respect to the $H$-actions on both sides (Remark 3.9), the uniqueness of an $H$-invariant holomorphic function implies that

$$
\operatorname{dim} \mathcal{S}_{\bar{\Omega}}^{\prime}(V, F(\lambda))^{H}=1 .
$$

Let $F(\lambda)$ be a spherical $H$-representation with highest weight $\lambda$ and $0 \neq v_{0} \in$ $F(\lambda)^{\mathfrak{h} \cap \mathfrak{k}}$. We have seen in the preceding section that there exists a unique $H$ invariant tempered distribution $R_{\lambda}^{H} \in \mathcal{S}_{\bar{\Omega}}^{\prime}(V, F(\lambda))$ with $\mathcal{F}\left(R_{\lambda}^{H}\right)(i e)=v_{0}$.

Lemma 5.3. The prescription

$$
\nu: N(\lambda) \cong e^{-\operatorname{tr}} \operatorname{Pol}(V, F(\lambda)) \rightarrow \mathbb{C}, \quad e^{-\operatorname{tr}} f \mapsto\left\langle R_{\lambda}^{H}, e^{-\operatorname{tr}} f\right\rangle
$$

is a non-zero element of $\left(N(\lambda)^{\sharp}\right)^{\mathfrak{h}}$.

Proof. It follows from [17, Lemma B.1] that $\nu$ is well defined because $\left.e^{- \text {tr }}\right|_{\Omega}$ extends to a Schwartz function on $V$. Finally $\nu$ is annihilitad by $\mathfrak{h}$ since $R_{\lambda}^{H}$ is $H$-fixed.

Corollary 5.4. $L(\lambda)$ is spherical if and only if $\left\langle R_{\lambda}^{H}, J(\lambda)\right\rangle=\{0\}$.

The classification of unitary highest weight modules with spherical $F(\lambda)$. Let $\gamma_{1}, \ldots, \gamma_{r} \in \widehat{\Delta}_{n}^{+}$be a maximal system of orthogonal roots, where $\gamma_{r}$ is the highest root and $\gamma_{j-1}$ is maximal among all positive non-compact roots orthogonal to $\gamma_{j}, \ldots, \gamma_{r}$. Then a linear functional $\lambda \in i t^{*}$ is invariant under $(-\tau)$ if and only if $\lambda \in \operatorname{span}\left\{\gamma_{j}: j=1, \ldots, r\right\}$ because the real rank of $\mathfrak{g}$ equals $r$ (cf. Definition $3.3(\mathrm{~b}))$. Therefore it is of the form

$$
\lambda=\sum_{j=1}^{r} \frac{\lambda\left(\check{\gamma}_{j}\right)}{2} \gamma_{j} .
$$

Since $\left.\gamma_{j} \circ c_{\mathfrak{g}}\right|_{\mathfrak{b}}=\varepsilon_{j} \in \mathfrak{b}^{*}$, we have

$$
\lambda_{\mathfrak{b}}:=\left.\lambda \circ c_{\mathfrak{g}}\right|_{\mathfrak{b}}=\sum_{j=1}^{r} \frac{\lambda\left(\check{\gamma}_{j}\right)}{2} \varepsilon_{j} .
$$

That $F(\lambda)$ is an $\mathfrak{h} \cap \mathfrak{k}$-spherical highest weight module of $\mathfrak{k}$ is equivalent to $-\tau . \lambda=$ $\lambda$ and the condition that $\lambda_{\mathfrak{b}} \in \mathfrak{b}^{*}$ is the highest weight of a spherical representation of $\mathfrak{h}$ which is equivalent to

$$
\lambda\left(\check{\gamma}_{j}\right)-\lambda\left(\check{\gamma}_{i}\right) \in 2 \mathbb{N}_{0} \quad \text { for } \quad i<j
$$

(cf. Definition 3.3(a)). For $\lambda=\lambda^{u}$ as in (5.1) this means that $m_{j}=\lambda\left(\check{\gamma}_{j}\right)$.

The following theorem shows that the classification of unitary highest weight modules with spherical $F(\lambda)$ is much simpler than the general case.

Theorem 5.5. If $F\left(\lambda^{0}\right)$ is $\mathfrak{h} \cap \mathfrak{k}$-spherical, $\lambda_{\mathfrak{b}}=\sum_{j=1}^{r} \frac{m_{j}}{2} \varepsilon_{j}$, and $k \in\{0, \ldots, r-1\}$ is determined by $m_{1} \leq \ldots \leq m_{k}<m_{k+1}=\ldots=m_{r}$, then

$$
\left.l\left(\lambda^{0}\right)=\left\{k d,(2 k+1) \frac{d}{2}, \ldots,(k+r-1) \frac{d}{2}\right\} \dot{\cup}\right](k+r-1) \frac{d}{2}, \infty[.
$$


Proof. First [8, 6.6] implies that $\left.l\left(\lambda^{0}\right)=\left\{u_{0}, \ldots, u_{m}\right\} \dot{\cup}\right] u_{m}, \infty[$ with $m=r-k-1$, so that it remains to determine $u_{0}$.

Let $u:=u_{0}$. To compute this parameter, we recall from 8 that $\leq(\lambda)=1$, and that the highest weight of $J(\lambda)$ is given by the unique dominant integral functional $\mu_{0}$ conjugate to $\lambda-\gamma_{r}$ under the Weyl group of $\widehat{\Delta}_{k}$. Since in our context $\lambda$ and $\gamma_{r}$ can be identified with elements of $\mathfrak{b}^{*}$ and the Weyl group contains in particular the permutations $\sigma_{i j}$ for two element $\gamma_{i}$ and $\gamma_{j}$, it follows that

$$
\mu_{0}=\sigma_{r, k+1} \cdot\left(\lambda-\gamma_{r}\right)=\lambda-\gamma_{k+1} .
$$

That this functional is dominant is a consequence of the fact that $m_{k+1}-2 \geq m_{k}$ which follows from (5.2).

To calculate $u_{0}$, we use the fact that $L\left(\lambda^{u_{0}}\right)$ and $L\left(\mu_{0}\right)$ correspond to the same eigenvalues of the Casimir operator of $\mathfrak{g}$, which implies that

$$
\|\lambda+\widehat{\rho}\|^{2}=\left\|\mu_{0}+\widehat{\rho}\right\|^{2}=\left\|\lambda+\widehat{\rho}-\gamma_{k+1}\right\|^{2}
$$

for $\widehat{\rho}:=\frac{1}{2} \sum_{\widehat{\alpha} \in \widehat{\Delta}^{+}} \widehat{\alpha}$. This means that $2\left\langle\lambda+\widehat{\rho}, \gamma_{k+1}\right\rangle=\left\|\gamma_{k+1}\right\|^{2}$ which is the same as

$$
(\lambda+\widehat{\rho})\left(\check{\gamma}_{k+1}\right)=1 .
$$

Since $\lambda\left(\check{\gamma}_{k+1}\right)=\lambda\left(\check{\gamma}_{r}\right)=-u_{0}$, we obtain $u_{0}=\widehat{\rho}\left(\check{\gamma}_{k+1}\right)-1$.

To compute this number, we first observe that it equals one half of the trace of $\check{\gamma}_{k+1}$ on the positive root spaces in $\mathfrak{g}_{\mathbb{C}}$. Since $\check{\gamma}_{k+1}$ corresponds to the element $2 L\left(c_{k+1}\right)$ under the Cayley transform, the contribution of the non-compact root spaces is given by

$$
\operatorname{tr}_{V} L\left(c_{k+1}\right)=(r-1) \frac{d}{2}+1
$$

(this is a consequence of the Peirce decomposition of $V$; see [11, Th. IV.2.1]). The contribution of the compact roots equals

$$
\begin{aligned}
\frac{1}{2} \sum_{\alpha \in \Sigma^{+}}\left(\operatorname{dim} \mathfrak{h}^{\alpha}\right) \alpha\left(2 L\left(c_{k+1}\right)\right) & =\frac{d}{2} \sum_{i<j}\left(\varepsilon_{j}-\varepsilon_{i}\right)\left(L\left(c_{k+1}\right)\right) \\
& =\frac{d}{2}(k-(r-k-1))=(2 k-r+1) \frac{d}{2} .
\end{aligned}
$$

Summing up, we obtain

$$
u_{0}=\widehat{\rho}\left(\check{\gamma}_{k+1}\right)-1=(r-1+2 k-r+1) \frac{d}{2}=k d .
$$

\section{Support properties.}

Lemma 5.6. Let $D \in \mathcal{S}^{\prime}(V)$ be a tempered distribution whose support is contained in $\bar{\Omega}$. Further let $I \subseteq \operatorname{Pol}(V)$ be an ideal such that $\left\langle D, e^{-\operatorname{tr}} I\right\rangle=\{0\}$. Then

$$
\operatorname{supp}(D) \subseteq\{x \in V:(\forall f \in I) f(x)=0\} .
$$

Proof. Since $D$ is supported by $\bar{\Omega}$, the product $D e^{-\operatorname{tr}}$ defines a tempered distribution on $V$ (cf. [17, Lemma B.1]). Let $f \in I$. We claim that $f D=0$. For all polynomials $p \in \operatorname{Pol}(V)$ we have

$$
\left\langle f D, e^{-\operatorname{tr}} p\right\rangle=\left\langle D, e^{-\operatorname{tr}} p f\right\rangle=0 .
$$


On the other hand, the Fourier transform $\mathcal{F}(f D) \in \operatorname{Hol}\left(T_{\Omega}\right)$ (cf. Section 2) satisfies

$$
\left(p\left(\frac{\partial}{\partial z}\right) \mathcal{F}(f D)\right)(i e)=\left\langle f D, e^{-\operatorname{tr}} p(i \cdot)\right\rangle=0 .
$$

Since $\mathcal{F}(f D)$ is a holomorphic function on $T_{\Omega}$ and $p$ was arbitrary, we conclude that $\mathcal{F}(f D)=0$, and hence that $f D=0$. This implies that $\operatorname{supp}(D) \subseteq f^{-1}(0)$ (17, Lemma B.4]), hence yields the lemma.

Lemma 5.7. Let $D \in \mathcal{S}^{\prime}(V) \otimes F(\lambda)$ be a tempered distribution whose support is contained in $\bar{\Omega}$. If

$$
\left\langle D, e^{-\operatorname{tr}} I_{k} \otimes F(\lambda)\right\rangle=\{0\},
$$

then $\operatorname{supp}(D) \subseteq \mathcal{O}_{k}$.

Proof. Let $\alpha \in F(\lambda)^{*}$. Our assumption implies that $\left\langle\alpha \circ D, e^{-\operatorname{tr}} I_{k}\right\rangle=\{0\}$, so that Lemma 5.6 implies that then $\operatorname{supp}(\alpha \circ D) \subseteq \mathcal{O}_{k}$. Since $\alpha$ was arbitrary, the assertion follows.

Proposition 5.8. If $L(\lambda)$ is unitarizable and spherical, then

$$
\operatorname{supp}\left(R_{\lambda}^{H}\right) \subseteq \operatorname{supp}\left(R_{\lambda}\right) .
$$

Proof. If $\operatorname{supp}\left(R_{\lambda}\right)=\bar{\Omega}$, there is nothing to show. So we may assume that $u=k \frac{d}{2}$ and $\operatorname{supp}\left(R_{\lambda}\right)=\overline{\mathcal{O}_{k}}$ (Theorem 4.4). Then

$$
e^{-\operatorname{tr}} I_{k} \otimes F(\lambda) \subseteq J(\lambda),
$$

so that the assumption that $L(\lambda)$ is spherical implies that $R_{\lambda}^{H}$ vanishes on this space. Hence the assertion follows from Lemma 5.7.

As the preceding proposition suggests, information on the support of $R_{\lambda}^{H}$ might provide information on whether $L(\lambda)$ is spherical or not.

Proposition 5.9. About the support of the distributions $R_{\lambda}^{H}, \lambda=\lambda^{s}$, we have the following information:

1. If $s=j d, j \in\{k, \ldots, r-1\}$, then $R_{\lambda}^{H}$ is a measure supported by $\overline{\mathcal{O}_{k}}$ for which $\overline{\mathcal{O}_{k-1}}$ is zero set.

2. If $s>(r-1) d$, then $R_{\lambda}^{H}$ is a measure supported by $\bar{\Omega}$ for which $\partial \Omega$ is a zero set.

3. If $s \notin\{0, \ldots,(r-1) d\}-2 \mathbb{N}_{0}$ and $s \leq(r-1) d$, then $R_{\lambda}^{H}$ is not a measure and its support is all of $\bar{\Omega}$.

4. If $s=j d-2 m$ with $m \in \mathbb{N}$ and $j \in\{k, \ldots, r-1\}$, then $\operatorname{supp}\left(R_{\lambda}^{H}\right) \subseteq \overline{\mathcal{O}_{j}}$.

Proof. 1. follows directly from Theorem 5.1 and [17, Prop. III.6].

2. This is a consequence of the fact that the density of $R_{\lambda}^{H}$ with respect to the $H$-semi-invariant measure $R_{\frac{s}{2}}$ on $\Omega$ does not vanish anywhere.

3 . In view of the relation

$$
\Delta^{l} R_{s}=(s)_{l} R_{s+l}, \quad s \in \mathbb{C}, l \in \mathbb{N}
$$

([17, Prop. III.1]), we obtain for $s>(r-1) d$ the relation

$$
\begin{aligned}
\Delta^{l} R_{\lambda^{s}}^{H} & =\left(\frac{s}{2}\right)_{-\mathbf{m}}^{-1} \Delta^{l} \eta R_{\frac{s}{2}}=\left(\frac{s}{2}\right)_{-\mathbf{m}}^{-1}\left(\frac{s}{2}\right)_{l} \eta R_{\frac{s+2 l}{2}} \\
& =\left(\frac{s}{2}\right)_{-\mathbf{m}}^{-1}\left(\frac{s}{2}\right)_{l}\left(\frac{s+2 l}{2}\right)_{-\mathbf{m}} R_{\lambda^{s+2 l}}^{H}=\left(\frac{s}{2}-\mathbf{m}\right)_{l} R_{\lambda^{s+2 l}}^{H} .
\end{aligned}
$$


Since both sides are weakly holomorphic functions with values in $\mathcal{S}^{\prime}\left(V, F_{0}\right)$, we conclude that

$$
\Delta^{l} R_{\lambda^{s}}^{H}=\left(\frac{s}{2}-\mathbf{m}\right){ }_{l} R_{\lambda^{s+2 l}}^{H}
$$

holds for all $s \in \mathbb{C}$. Now the hypotheses shows that $\left(\frac{s}{2}-\mathbf{m}\right)_{l} \neq 0$ for any $l \in \mathbb{N}_{0}$. Therefore 1 and 2 together with (5.3) imply that $\bar{\Omega} \subseteq \operatorname{supp}\left(R_{\lambda^{s}}^{H}\right)$, hence equality.

4. From the proof of [17, Prop. V.2] we get

$$
\Delta\left(\frac{\partial}{\partial x}\right) R_{\lambda^{s}}^{H}=R_{\lambda^{s-2}}^{H},
$$

so that 4 follows from 1 .

The situation is particularly simple for $\lambda^{0}=0$, where $k=0$, so that Proposition 5.9 gives complete information on the support of $R_{\lambda^{s}}^{H}=R_{\frac{s}{2}}$. Fortunately the classification in Theorem 5.5 shows that the information from Proposition 5.9 suffices to deal with all those distributions $R_{\lambda}^{H}$ for which $F(\lambda)$ is spherical and $L(\lambda)$ is unitary. This is made precise by the following result:

Proposition 5.10. Suppose that $F\left(\lambda^{0}\right)$ is $\mathfrak{h} \cap \mathfrak{k}$-spherical and that $L(\lambda)$ is singular and unitary. Then the following assertions hold:

1. $R_{\lambda}^{H}$ is a measure if and only if $\leq(\lambda)$ is odd.

2. If $u=j \frac{d}{2}$ with $j \leq r-1$ odd, then $R_{\lambda}^{H}$ does not vanish on $e^{-\operatorname{tr}} I_{j} \otimes F(\lambda)$.

Proof. 1. Let $\lambda=\lambda^{u_{j}}$. Then $\leq(\lambda)=j+1$ and $\frac{u_{j}}{2}=\left(k+\frac{j}{2}\right) \frac{d}{2}$ (Theorem 5.5). Since

$$
\frac{u_{j}}{2} \leq\left(k+\frac{r-k-1}{2}\right) \frac{d}{2}=\frac{r+k-1}{2} \frac{d}{2} \leq(r-1) \frac{d}{2},
$$

the assertion follows from Proposition 5.9.1.

2. The ideal $I_{j}$ contains in particular the polynomial $\Delta_{\mathbf{j}+\mathbf{1}}$ for

$$
\mathbf{j}+\mathbf{1}=(\underbrace{1, \ldots, 1}_{(j+1)-\text { times }}, 0, \ldots, 0),
$$

so that it suffices to show that

$$
\left\langle R_{\lambda}^{H}, e^{-\operatorname{tr}} \Delta_{\mathbf{j}+\mathbf{1}} v_{\lambda}\right\rangle \neq 0,
$$

where $v_{\lambda} \in F(\lambda)$ is a real highest weight vector for $\mathfrak{h}$ with highest weight $\lambda_{\mathfrak{b}}=$ $\sum_{i=1}^{r} \frac{m_{i}-u}{2} \varepsilon_{i}$.

Let $\mathbf{s}:=\left(s_{1}, \ldots, s_{r}\right) \in \mathbb{C}^{r}$ and let $R_{\mathbf{s}} \in \mathcal{S}_{\bar{\Omega}}(\mathbb{C})$ be the multiparameter Riesz distribution which is uniquely determined by

$$
\mathcal{F}\left(R_{\mathbf{s}}\right)(i x)=\Delta_{\mathbf{s}}\left(x^{-1}\right) \quad \text { for } \quad x \in \Omega
$$

(cf. [11, Ch. VII]). Now [17, Lemma V.4] yields

$$
\left\langle R_{\lambda}^{H}, v_{\lambda}\right\rangle=\left\langle v_{0}, v_{\lambda}\right\rangle R_{\frac{u-\mathbf{m}}{2}} .
$$

On the other hand we have

$$
\Delta_{\mathbf{j}+\mathbf{1}} \cdot R_{\frac{u-\mathbf{m}}{2}}=\left(\frac{u-\mathbf{m}}{2}\right)_{\mathbf{j}+\mathbf{1}} R_{\frac{u-\mathbf{m}}{2}}+\mathbf{j}+\mathbf{1}
$$

with

$$
\left(\frac{u-\mathbf{m}}{2}\right)_{\mathbf{j}+\mathbf{1}}=\prod_{l=1}^{j+1}\left(\frac{u-m_{l}}{2}-(l-1) \frac{d}{2}\right)
$$


([17, Prop. III.1(iii)]). This eventually leads to

$$
\begin{aligned}
\left\langle R_{\lambda}^{H}, e^{-\operatorname{tr}} \Delta_{\mathbf{j}+\mathbf{1}} v_{\lambda}\right\rangle & =\mathcal{F}\left(\Delta_{\mathbf{j}+\mathbf{1}}\left\langle R_{\lambda}^{H}, v_{\lambda}\right\rangle\right)(-i e)=\left(\frac{u-\mathbf{m}}{2}\right)_{\mathbf{j}+\mathbf{1}} \Delta_{\frac{u-\mathbf{m}}{2}+\mathbf{j}+\mathbf{1}}(e)\left\langle v_{0}, v_{\lambda}\right\rangle \\
& =\left(\frac{u-\mathbf{m}}{2}\right)_{\mathbf{j}+\mathbf{1}}\left\langle v_{0}, v_{\lambda}\right\rangle .
\end{aligned}
$$

Since $\left\langle v_{0}, v_{\lambda}\right\rangle \neq 0$ (cf. [17 Lemma II.1]), it remains to show that $\left(\frac{u-\mathbf{m}}{2}\right)_{\mathbf{j}+\mathbf{1}}$ does not vanish if $j$ is odd. In view of $m_{l}=0$ for $l>k$, we have

$$
\prod_{l=k+1}^{j+1}\left(\frac{u-m_{l}}{2}-(l-1) \frac{d}{2}\right)=\prod_{l=k+1}^{j+1} \frac{d}{2}\left(\frac{j}{2}-(l-1)\right) \neq 0 .
$$

For $l \leq k$ we use $j \geq 2 k$ (Theorem 5.5) to obtain

$$
-m_{l}+\frac{u}{2}-(l-1) \frac{d}{2} \geq \frac{u}{2}-(l-1) \frac{d}{2} \geq \frac{d}{2}(k-(l-1))>0 .
$$

Therefore all factors in $\left(\frac{u-\mathbf{m}}{2}\right) \mathbf{j}+\mathbf{1}$ are non-zero.

Theorem 5.11 (Classification for singular supports). Let $G$ be a simply connected hermitian Lie group of tube type and $G / H$ an associated symmetric space of Cayley type. Suppose that $F(\lambda)$ is $\mathfrak{h} \cap \mathfrak{k}$-spherical and that $L(\lambda)$ is unitary with $\operatorname{supp}\left(R_{\lambda}\right) \neq$ $\bar{\Omega}$. Then $L(\lambda)$ is spherical if and only if $\leq(\lambda)$ is odd.

Proof. Since $\operatorname{supp}\left(R_{\lambda}\right)$ is smaller than $\bar{\Omega}$, we have $u=j \frac{d}{2}$ for some $j \leq r-1$ (Theorem 4.4). Moreover, in view of Remark 2.3, Theorem 5.5 shows that $\leq(\lambda)$ is odd if and only if $j$ is even. If this is the case, then $L(\lambda)$ is spherical by Theorem 2.8 . If $j$ is odd, then Proposition 5.10 shows that $R_{\lambda}^{H}$ does not vanish on $e^{-\operatorname{tr}} I_{j} \otimes F(\lambda)$ which is contained in $J(\lambda)$ (Proposition 4.6). Therefore Corollary 5.4 implies that $L(\lambda)$ is not spherical.

Corollary 5.12 (Classification for the scalar case). If $L(\lambda)$ is singular, unitary and of scalar type, then $L(\lambda)$ is spherical if and only if $\leq(\lambda)$ is odd.

Proof. In the scalar case $F(\lambda)$ is always $H \cap K$-spherical and $\lambda$ is singular if and only $\operatorname{supp}\left(R_{\lambda}\right) \neq \bar{\Omega}$. Therefore the assertion follows from Theorem 5.11.

Lemma 5.13. If $L(\lambda)$ and $L(\mu)$ are unitary and spherical, then the same holds for $L(\lambda+\mu)$.

Proof. Since $L(\lambda)$ and $L(\mu)$ are unitary, the highest weight module $L(\lambda+\mu)$ can be embedded into the unitary module $L(\lambda) \otimes L(\mu)$ as the submodule generated by $v_{\lambda} \otimes v_{\mu}$, where $v_{\lambda}$ and $v_{\mu}$ are highest weight vectors ([26, Prop. IX.1.15]). It follows in particular that $L(\lambda+\mu)$ is unitary. Moreover, if $\nu_{\lambda} \in\left(L(\lambda)^{\sharp}\right)^{\mathfrak{h}}$ and $\nu_{\mu} \in\left(L(\mu)^{\sharp}\right)^{\mathfrak{h}}$ are non-trivial $\mathfrak{h}$-invariant antilinear functionals, then $\nu_{\lambda} \otimes \nu_{\mu}$ is an $\mathfrak{h}$-invariant antilinear functional on $L(\lambda) \otimes L(\mu)$ with

$$
\left(\nu_{\lambda} \otimes \nu_{\mu}\right)\left(v_{\lambda} \otimes v_{\mu}\right)=\nu_{\lambda}\left(v_{\lambda}\right) \nu_{\mu}\left(v_{\mu}\right) \neq 0
$$

(Proposition 1.5). This shows that the restriction of $\nu_{\lambda} \otimes \nu_{\mu}$ to $L(\lambda+\mu)$ is non-zero and hence that this module is spherical. 
Remark 5.14. Let $u_{0}=k d$ as in Theorem 5.5. Then Theorem 5.11 shows that $L(\lambda)$ is spherical for $u=u_{j}=k d+j \frac{d}{2}$ if $j$ is even (which corresponds to odd reduction level) and not spherical if $j$ is odd, provided $u_{j} \leq(r-1) \frac{d}{2}$. This means that

$$
j \leq r-1-2 k \text {. }
$$

For an odd $j \in\{r-2 k, \ldots, r-k-1\}$ Theorem 5.11 gives no information.

Suppose that for some $j$ the module $L\left(\lambda^{u_{j}}\right)$ is spherical. We know from Corollary 5.12 that $L(d \zeta)$ is spherical, so that Lemma 5.13 and

$$
\lambda^{u_{j+2}}=\lambda^{u_{j}}+d \zeta
$$

imply that $L\left(\lambda^{u_{j+2}}\right)$ is spherical. This argument can be iterated, and it shows that if for one odd value of $j$ the module $L\left(\lambda^{u_{j}}\right)$ is spherical, then it is true for all larger (odd) values of $j$.

We have already seen in Corollary 5.12 that for singular support of $R_{\lambda}$ and even reduction level the scalar type highest weight modules are not spherical. It is instructive to observe that this in general not ruled out by the support condition in Proposition 5.8. The following proposition shows that the support behavior is actually quite complicated.

Proposition 5.15. For $s>(r-1) \frac{d}{2}$ we always have $\operatorname{supp}\left(R_{\frac{s}{2}}\right) \subseteq \operatorname{supp}\left(R_{s}\right)$. For $s=\frac{k d}{2}, k \in\{0,1, \ldots, r-1\}$ we have:

1. If $k$ is even, then $\operatorname{supp}\left(R_{\frac{s}{2}}\right) \subseteq \operatorname{supp}\left(R_{s}\right)$.

2. If $k$ is odd, then we have:

(a) If $d=1,2$, then $\operatorname{supp}\left(R_{\frac{s}{2}}\right) \nsubseteq \operatorname{supp}\left(R_{s}\right)$.

(b) If $d=4$, then $\operatorname{supp}\left(R_{\frac{s}{2}}\right) \subseteq \operatorname{supp}\left(R_{s}\right)$.

(c) If $d=8$ and $r=3$, then $\operatorname{supp}\left(R_{\frac{s}{2}}\right) \subseteq \operatorname{supp}\left(R_{s}\right)$.

(d) If $d=n-2$ and $r=2$, then $\operatorname{supp}\left(R_{\frac{s}{2}}\right) \subseteq \operatorname{supp}\left(R_{s}\right)$ if and only if $n \equiv 2 \bmod 4$.

Proof. 1. This follows from $\operatorname{supp}\left(R_{\frac{s}{2}}\right)=\overline{\mathcal{O}_{\frac{k}{2}}} \subseteq \overline{\mathcal{O}_{k}}=\operatorname{supp}\left(R_{s}\right)$.

2. (1) $d=1$ : Then

$$
\frac{k d}{4}=\frac{k}{4} \notin\left\{j \frac{d}{2}: j=0, \ldots, r-1\right\}-\mathbb{N}_{0}
$$

because $k \not \equiv 2 j \bmod 4$ for each $j$. Hence Proposition 5.8 implies that

$$
\operatorname{supp}\left(R_{\frac{s}{2}}\right)=\bar{\Omega} \not \subset \operatorname{supp}\left(R_{s}\right)
$$

in this case.

$d=2$ : If $k$ is odd, then

$$
\frac{k d}{4}=\frac{k}{2} \notin\left\{j \frac{d}{2}: j=0, \ldots, r-1\right\}-\mathbb{N}_{0}=\{j: j=0, \ldots, r-1\}-\mathbb{N}_{0}
$$

because $\frac{k}{2}$ is not an integer.

(2) $d=4$ : If $k$ is odd, then

$$
\frac{k d}{4}=k \in\left\{j \frac{d}{2}: j=0, \ldots, r-1\right\}-\mathbb{N}_{0}=\{2 j: j=0, \ldots, r-1\}-\mathbb{N}_{0}
$$

and the minimal $j$ with $k \in 2 j-\mathbb{N}_{0}$ is given by $j=\frac{k+1}{2} \leq k$. We conclude that in this case we always have $\operatorname{supp}\left(R_{\frac{s}{2}}\right) \subseteq \overline{\mathcal{O}_{j}} \subseteq \overline{\mathcal{O}_{k}}=\operatorname{supp}\left(R_{s}\right)$. 
(3) $d=8$ and $r=3$ : Then $k$ odd implies that $k=1$. Now

$$
\frac{k d}{4}=2=4 \cdot 1-2 \in\left\{j \frac{d}{2}: j=0, \ldots, r-1\right\}-\mathbb{N}_{0}=\{4 j: j=0,1,2\}-\mathbb{N}_{0} .
$$

Hence $\operatorname{supp}\left(R_{\frac{s}{2}}\right) \subseteq \overline{\mathcal{O}_{1}}=\operatorname{supp}\left(R_{s}\right)$.

(4) $d=n-2$ and $r=2$ : Then $k=1$ and therefore

$$
\begin{aligned}
\frac{k d}{4} & =\frac{n-2}{4} \in\left\{j \frac{d}{2}: j=0, \ldots, r-1\right\}-\mathbb{N}_{0} \\
& =\left\{j \frac{d}{2}: j=0,1\right\}-\mathbb{N}_{0}=\left\{0, \frac{n-2}{2}\right\}-\mathbb{N}_{0}
\end{aligned}
$$

is equivalent to

$$
\frac{n-2}{2}-\frac{n-2}{4}=\frac{n-2}{4} \in \mathbb{N} .
$$

Thus $\operatorname{supp}\left(R_{\frac{s}{2}}\right) \nsubseteq \operatorname{supp}\left(R_{s}\right)$ for $n \not \equiv 2 \bmod 4$, and for $n \equiv 2 \bmod 4$ we have $\operatorname{supp}\left(R_{\frac{s}{2}}\right) \subseteq \overline{\mathcal{O}_{1}}=\operatorname{supp}\left(R_{s}\right)$.

Example 5.16. We consider the Lie algebra $\mathfrak{g}=\mathfrak{s u}(r, r)$ corresponding to the Jordan algebra $V=\operatorname{Herm}(r, \mathbb{C})$. Here $\mathfrak{h} \cong \mathbb{R} \oplus \mathfrak{s l}(r, \mathbb{C})$, which we consider as a quotient of $\mathfrak{g l}(r, \mathbb{C})$ modulo $i \mathbb{R}$. This is most natural because $\mathfrak{g l}(r, \mathbb{C})$ acts naturally on $V$ by $X . A=X A+A X^{*}$, and the kernel of this representation is $i \mathbb{R}$. The complexification of $\mathfrak{h}$ is

$$
\mathfrak{h}_{\mathbb{C}} \cong \mathbb{C} \oplus \mathfrak{s l}(r, \mathbb{C}) \oplus \mathfrak{s l}(r, \mathbb{C})
$$

which we consider as a quotient of $\mathfrak{g l}(r, \mathbb{C}) \oplus \mathfrak{g l}(r, \mathbb{C})$ acting on $V_{\mathbb{C}} \cong \operatorname{End}\left(\mathbb{C}^{r}\right)$ by $\left(X_{1}, X_{2}\right) . A=X_{1} A+A X_{2}^{*}$. Accordingly we write the highest weights of simple $\mathfrak{h}_{\mathbb{C}}$ modules as

$$
\lambda=\left(\lambda_{1}, \ldots, \lambda_{2 r}\right)=\left(\lambda^{+}, \lambda^{-}\right)
$$

with respect to the positive system

$$
\widehat{\Delta}_{k}^{+}=\left\{\varepsilon_{i}-\varepsilon_{j}: 1 \leq i<j \leq r ; r+1 \leq i<j \leq 2 r\right\}
$$

of roots of $\mathfrak{s l}(r, \mathbb{C}) \oplus \mathfrak{s l}(r, \mathbb{C})$.

The corresponding representation space $F(\lambda)$ has the structure

$$
F(\lambda)=F\left(\lambda^{+}\right) \otimes F\left(\lambda^{-}\right)=F\left(\lambda^{+}\right) \otimes F\left(\tilde{\lambda}^{-}\right)^{*} \cong \operatorname{Hom}\left(F\left(\tilde{\lambda}^{-}\right), F\left(\lambda^{+}\right)\right),
$$

where $\widetilde{\lambda}^{-}=\left(-\lambda_{2 r}, \ldots,-\lambda_{r+1}\right)$. The action of $\mathfrak{h}$ on this space is given by

$$
X . A=\pi_{\lambda^{+}}(X) A+A \pi_{\tilde{\lambda}^{-}}(X)^{*} .
$$

Therefore a fixed vector for $\mathfrak{h} \cap \mathfrak{k} \cong \mathfrak{s u}(r, \mathbb{C})$ corresponds to an intertwining operator for $\mathfrak{s u}(r, \mathbb{C})$, and hence for $\mathfrak{s l}(r, \mathbb{C})$, in $\operatorname{Hom}\left(F\left(\widetilde{\lambda}^{-}\right), F\left(\lambda^{+}\right)\right)$. This means that $F(\lambda)$ is spherical if and only if $F\left(\lambda^{+}\right) \cong F\left(\widetilde{\lambda}^{-}\right)$as $\mathfrak{s l}(r, \mathbb{C})$-modules. With $\mu:=\lambda^{+}$we therefore get $F(\lambda) \cong \operatorname{End}(F(\mu))$ with $\mathfrak{s l}(r, \mathbb{C})$ acting by $X . A=\pi_{\mu}(X) A+A \pi_{\mu}(X)^{*}$.

The restricted highest weight of this representation with respect to the subspace $\mathfrak{b}$ of real diagonal elements in $\mathfrak{h}$ is given by

$$
\lambda_{\mathfrak{b}}=\sum_{j=1}^{r} \frac{m_{j}}{2} \varepsilon_{j}=\sum_{j=1}^{r} \frac{\lambda_{r+1-j}-\lambda_{r+j}}{2} \varepsilon_{j} .
$$


The normalization $m_{r}=0$ leads to $\lambda_{1}=\lambda_{2 r}$, and for the spherical representations further to

$$
\lambda_{\mathfrak{b}}=\sum_{j=1}^{r} \lambda_{r+1-j} \varepsilon_{j}
$$

because $\lambda^{+}=-\widetilde{\lambda}^{-}$in this case. We determine $k \in\{0, \ldots, r-1\}$ by $\lambda_{1}=\lambda_{2}=$ $\ldots=\lambda_{r-k}>\lambda_{r-k+1}$. Then Theorem 5.5 implies that $m=r-1-k$ and $u_{0}=2 k$ because $d=2$ holds for $\mathfrak{g}=\mathfrak{s u}(r, r)$.

It is interesting to observe that the relation $F(\lambda) \cong \operatorname{End}(F(\mu))$ as representations of $H$ implies that $\tau_{\mu} \circ \widetilde{P}: T_{\Omega} \rightarrow \operatorname{End}(F(\mu))$ is $H$-equivariant, so that the injectivity of the Fourier transform implies for $v_{0}=\mathbf{1} \in \operatorname{End}(F(\mu))$ the relation

$$
R_{\mu}=R_{\lambda}^{H}
$$

where $F(\mu)$ is interpreted as a module of $\mathfrak{h}_{\mathbb{C}}$ which is trivial on the second $\mathfrak{s l}(r, \mathbb{C})$ factor. It has been shown by J. L. Clerc in [5] that for these representations $L(\mu)$ is unitary if and only if $\frac{u}{2} \in\{k, k+1, \ldots, r-1\}$ or $u>(r-1)$. According to Proposition 5.8, these are the cases where $R_{\mu}=R_{\lambda}^{H}$ is a measure, and they correspond to the cases with odd reduction level. Then the unitary $\mathfrak{g}$-module

$$
L(\mu) \otimes L(\mu)^{*} \subseteq \operatorname{End}(L(\mu))
$$

contains a $\mathfrak{g}$-submodule isomorphic to $L(\lambda)$, and the trace is an $\mathfrak{h}$-invariant linear functional on $L(\lambda)$. This construction can also be used to prove that the representations $L(\lambda)$ are spherical for odd reduction levels.

Our results in this section suggest that for even reduction level a singular highest weight representation is never spherical. At least we do not know of any counterexample.

Similarly, in all cases of singular representation where we could decide whether $L(\lambda)$ is spherical or not, this happens if and only if the distribution $R_{\lambda}^{H}$ is a measure. In the scalar case this follows from Proposition 5.9 and Theorem 5.5.

\section{APPENDIX: Regularity of THE HOLOMORPHIC DISCRETE SERIES}

In this appendix we show that the highest weight modules $L(\lambda)$ corresponding to the discrete series of a compactly causal symmetric space $G / H$ are regular in the sense that $N(\lambda) \cong L(\lambda)$. This shows in particular that these representations do not provide any further information on sphericalness of singular highest weight representations.

Let $\mathfrak{g}$ be a hermitian Lie algebra, $\mathfrak{t} \subseteq \mathfrak{g}$ a compactly embedded Cartan subalgebra and $\widehat{\Delta}=\widehat{\Delta}\left(\mathfrak{g}_{\mathbb{C}}, \mathfrak{t}_{\mathbb{C}}\right)$ the corresponding root system. We choose a positive system $\widehat{\Delta}^{+}$of $\widehat{\Delta}$ as in Section 1 and set $\widehat{\rho}:=\frac{1}{2} \sum_{\widehat{\alpha} \in \widehat{\Delta}^{+}} \widehat{\alpha}, \rho_{k}:=\frac{1}{2} \sum_{\widehat{\alpha} \in \widehat{\Delta}_{k}^{+}} \widehat{\alpha}$ and $\widehat{\rho}_{n}:=$ $\frac{1}{2} \sum_{\widehat{\alpha} \in \Delta_{n}^{+}} \widehat{\alpha}$.

If $\lambda \in i t^{*}$ is dominant integral w.r.t. $\widehat{\Delta}_{k}^{+}$, then the condition for $L(\lambda)$ to correspond to the relative holomorphic discrete series of $G$ is given by Harish-Chandra's condition (cf. [13])
$D S(G)$

$$
\left(\forall \widehat{\alpha} \in \widehat{\Delta}_{n}^{+}\right)
$$$$
\langle\lambda+\widehat{\rho}, \widehat{\alpha}\rangle<0 .
$$

Elementary algebraic considerations involving the Parthasarathy inequality (cf. [9] Prop. 3.9]) or [21, p. 35] imply that $\mathrm{DS}(\mathrm{G})$ implies that $\lambda$ is regular, i.e, $N(\lambda)=$ $L(\lambda)$. 
Now equip $\mathfrak{g}$ with an involution $\tau$ such that $(\mathfrak{g}, \tau)$ becomes compactly causal. Recall the root system $\Delta=\Delta\left(\mathfrak{g}^{c}, \mathfrak{a}\right)$. The fact that $\mathfrak{z}(\mathfrak{k}) \subseteq \mathfrak{q}$ implies that we can choose $\widehat{\Delta}^{+}$such that $-\tau \widehat{\Delta}^{+} \subseteq \widehat{\Delta}^{+} \cup \mathfrak{a}^{\perp}$. Then $\Delta^{+}:=\widehat{\Delta}^{+} \mid \mathfrak{a} \backslash\{0\}$ is a positive system of $\Delta$. Recall the definition of $\Delta_{n}^{+}$and $\Delta_{k}^{+}$from Section 1. Set $\rho:=\frac{1}{2} \sum_{\alpha \in \Delta^{+}} m_{\alpha} \alpha$, $\rho_{k}:=\frac{1}{2} \sum_{\alpha \in \Delta_{k}^{+}} m_{\alpha} \alpha$ and $\rho_{n}:=\frac{1}{2} \sum_{\alpha \in \Delta_{n}^{+}} m_{\alpha} \alpha$ with $m_{\alpha}=\operatorname{dim}\left(\mathfrak{g}^{c}\right)^{\alpha}$.

Let $p: \mathfrak{t}_{\mathbb{C}} \rightarrow \mathfrak{a}_{\mathbb{C}}$ denote the orthogonal projection with respect to the CartanKilling form. Then the adjoint map $p^{*}: \mathfrak{a}_{\mathbb{C}}^{*} \rightarrow \mathfrak{t}_{\mathbb{C}}^{*}$ is injective and via this inclusion mapping we identify in the sequel $\mathfrak{a}_{\mathbb{C}}^{*}$ with a subspace of $\mathfrak{t}_{\mathbb{C}}^{*}$.

For a $H \cap K$-spherical highest weight $\lambda \in \mathfrak{a}^{*} \subseteq i \mathfrak{t}^{*}$ the condition for $L(\lambda)$ to belong to the relative holomorphic discrete series of $G / H$ is given by

$$
D S(G / H) \quad\left(\forall \alpha \in \Delta_{n}^{+}\right) \quad\langle\lambda+\rho, \alpha\rangle<0
$$

(cf. 29], 19]; see also 24]). It follows easily from the fact that $\widehat{\Delta}^{+}$and $\Delta^{+}$are compatible that $D S(G / H)$ implies $D S(G)$. Conversely it was observed in [28] Lemma 7.4] that $D S(G)$ is a weaker condition than $D S(G / H)$ for $H \cap K$-spherical highest weights $\lambda$. Hence the following result is of interest.

Theorem A.1. Let $\lambda \in \mathfrak{a}^{*}$ be dominant integral for $\widehat{\Delta}_{k}^{+}$and assume that $D S(G / H)$ holds. Then $\lambda$ is regular, i.e., $L(\lambda)=N(\lambda)$.

We are going to give a simple algebraic proof of Theorem A.1 using the Parthasarathy inequality.

Lemma A.2. We have $\widehat{\rho}_{n}=\rho_{n}$.

Proof. This follows from $\widehat{\rho}_{n} \in i \mathfrak{z}(\mathfrak{k})^{*}, \mathfrak{z}(\mathfrak{k}) \subseteq \mathfrak{a}$ and $\left.\widehat{\Delta}_{n}^{+}\right|_{\mathfrak{a}} \subseteq \Delta^{+}$.

Lemma A.3. If $\lambda \in \mathfrak{a}^{*}$ and $\mu \in i \mathfrak{t}^{*}$, then

$$
\|\lambda+\widehat{\rho}\|^{2}-\|\mu+\widehat{\rho}\|^{2}=\|\lambda+\rho\|^{2}-\|\mu+\rho\|^{2}-2\left\langle\widehat{\rho}_{k}-\rho_{k}, \mu\right\rangle .
$$

Proof. By Lemma A.2 we have

$$
\begin{aligned}
& \|\lambda+\widehat{\rho}\|^{2}-\|\mu+\widehat{\rho}\|^{2}=\|\lambda+\rho+(\widehat{\rho}-\rho)\|^{2}-\|\mu+\rho+(\widehat{\rho}-\rho)\|^{2} \\
= & \left\|\lambda+\rho+\left(\widehat{\rho}_{k}-\rho_{k}\right)\right\|^{2}-\left\|\mu+\rho+\left(\widehat{\rho}_{k}-\rho_{k}\right)\right\|^{2} \\
= & \|\lambda+\rho\|^{2}+2\left\langle\lambda+\rho, \widehat{\rho}_{k}-\rho_{k}\right\rangle-\|\mu+\rho\|^{2}-2\left\langle\mu+\rho, \widehat{\rho}_{k}-\rho_{k}\right\rangle \\
= & \|\lambda+\rho\|^{2}-\|\mu+\rho\|^{2}-2\left\langle\mu, \widehat{\rho}_{k}-\rho_{k}\right\rangle,
\end{aligned}
$$

where the last equality comes from $\widehat{\rho}_{k}-\rho_{k} \in \mathfrak{a}^{\perp}$.

Lemma A.4. We have $\widehat{\rho}_{k}=\rho_{k}+\rho_{k}^{0}$ with $\rho_{k}^{0}:=\frac{1}{2} \sum_{\alpha \in \widehat{\Delta}_{k}^{+} \cap \mathfrak{a}^{\perp}} \alpha \in \mathfrak{a}^{\perp}$.

Proof. Let $\alpha \in \widehat{\Delta}_{k}^{+}$and rewrite $\alpha$ as $\alpha=\alpha_{\mathfrak{q}}+\alpha_{\mathfrak{h}}$ with $\alpha_{\mathfrak{q}}=\left.\alpha\right|_{\mathfrak{t} \cap \mathfrak{q}}$ and $\alpha_{\mathfrak{h}}=\left.\alpha\right|_{\mathfrak{t} \cap \mathfrak{h}}$. Assume that $\alpha_{\mathfrak{q}} \neq 0$. Then $(-\tau) \cdot \widehat{\Delta}_{k}^{+} \subseteq \widehat{\Delta}_{k}^{+} \cup \mathfrak{a}^{\perp}$ and the fact that $\widehat{\Delta}_{k}^{+}$and $\Delta_{k}^{+}$are compatible yield $-\tau . \alpha=\alpha_{\mathfrak{q}}-\alpha_{\mathfrak{h}} \in \widehat{\Delta}_{k}^{+}$. Hence we get

$$
\frac{1}{2} \sum_{\substack{\alpha \in \widehat{\Delta}_{k}^{+} \\ \alpha_{\mathfrak{q}} \neq 0}} \alpha=\rho_{k} .
$$

Now the assertion of the lemma follows from $\widehat{\rho}_{k}=\frac{1}{2} \sum_{\substack{\alpha \in \widehat{\Delta}_{k}^{+} \\ \alpha_{\mathfrak{q}} \neq 0}} \alpha+\rho_{k}^{0}$. 
Proof of Theorem A.1. In view of Parthasarathy's condition (cf. 9, Prop. 3.9]), we only have to check that

$$
\|\lambda+\widehat{\rho}\|^{2}-\|\mu+\widehat{\rho}\|^{2}<0
$$

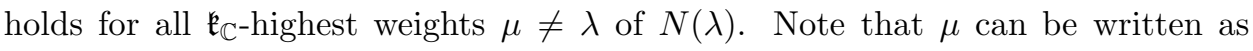
$\mu=\lambda-\sum_{\alpha \in \widehat{\Delta}_{n}^{+}} k_{\alpha} \alpha$ with $k_{\alpha} \in \mathbb{N}_{0}$. By Lemma A.2 we then have

$$
\begin{aligned}
\|\lambda+\widehat{\rho}\|^{2}-\|\mu+\widehat{\rho}\|^{2} & =\|\lambda+\rho\|^{2}-\|\mu+\rho\|^{2}-2\left\langle\widehat{\rho}_{k}-\rho_{k}, \mu\right\rangle \\
& =-\left\|\sum_{\alpha \in \widehat{\Delta}_{n}^{+}} k_{\alpha} \alpha\right\|^{2}+2\left(\sum_{\alpha \in \widehat{\Delta}_{n}^{+}} k_{\alpha}\langle\lambda+\rho, \alpha\rangle\right)-2\left\langle\widehat{\rho}_{k}-\rho_{k}, \mu\right\rangle .
\end{aligned}
$$

In the bottom line the first summand is clearly negative, the second one by assumption, and the non-positivity of the third one follows from Lemma A.4 since $\mu$ is dominant with respect to $\widehat{\Delta}_{k}^{+}$.

\section{REFERENCES}

[1] E. van den Ban, The principal series for a reductive symmetric space. I. H-fixed distribution vectors, Ann. Sci. École Norm. Sup. (4) 21:3 (1988), 359-412. MR 90a:22016

[2] E. van den Ban and P. Delorme, Quelques propriétés des représentations sphériques pour les espaces symétriques réductifs, J. Funct. Anal. 80 (1988), 284-307. MR 89j:22025

[3] F. Bien, D-modules and spherical representations, Princeton University Press, Princeton, NJ, 1990. MR 92f:22025

[4] J.L. Brylinski and P. Delorme, Vecteurs distributions H-Invariants pur les séries principales généralisées d'espaces symétriques reductifs et prolongement meromorphe d'intégrales d'Eisenstein, Invent. Math. 109 (1992), 619-664. MR 93m:22016

[5] J.-L. Clerc, Laplace transform and unitary highest weight modules, J. Lie Theory 5 (1995), 225-240. MR 97e:22014

[6] M.G. Davidson, T.J. Enright, and R.J. Stanke, Differential operators and highest weight representations, Memoirs of the Amer. Math. Soc. 94 (1991), 102 pp. MR 92c:22034

[7] H. Ding and K. Gross, Operator-valued Bessel functions on Jordan algebras, J. Reine Ang. Math. 435 (1993), 157-196. MR 93m:33010]

[8] T.J. Enright and A. Joseph, An intrinsic analysis of unitarizable highest weight modules, Math. Ann. 288 (1990), 571-594. MR 91m:17005

[9] T.J. Enright, R. Howe, and N. Wallach, A classification of unitary highest weight modules, Progr. Math., vol. 40, Birkhäuser Boston, Boston, MA, 1983, pp. 97-143. MR 86c:22028

[10] J. Faraut and A. Korányi, Function Spaces and Reproducing Kernels on Bounded Symmetric Domains, J. Funct. Anal. 88:1 (1990), 64-89. MR 90m:32049

[11] J. Faraut and A. Korányi, Analysis on Symmetric Cones, Oxford University Press, New York, 1994. MR 98g:17031

[12] M. Flensted-Jensen, Discrete series for semisimple symmetric spaces, Ann. of Math. 111:2 (1980), 253-311. MR 81h:22015

[13] Harish-Chandra, Representations of semi-simple Lie groups, VI, Amer. J. Math. 78 (1956), 564-628. MR 18:490d

[14] S. Helgason, Differential Geometry, Lie Groups, and Symmetric Spaces, Acad. Press, London, 1978. MR 80k:53081

[15] S. Helgason, Groups and Geometric Analysis, Acad. Press, London, 1984. MR 86c:22017. corrected reprint MR 2001h:22001

[16] J. Hilgert and B. Krötz, The Plancherel Theorem for invariant Hilbert spaces, Math. Z. 237(1) (2001), 61-83.

[17] J. Hilgert and K.-H. Neeb, Vector valued Riesz distributions on Euclidean Jordan algebras, J. Geom. Analysis 11 (1) (2001). CMP 2001:12

[18] J. Hilgert and G. Ólafsson, Causal Symmetric Spaces, Geometry and Harmonic Analysis, Acad. Press, London, 1997. MR 97m:43006

[19] J. Hilgert, G. Ólafsson, and B. Ørsted, Hardy Spaces on Affine Symmetric Spaces, J. Reine Angew. Math. 415 (1991), 189-218. MR 92h:22030 
[20] H.P. Jakobsen, Hermitian symmetric spaces and their unitary highest weight modules, J. Funct. Anal. 52 (1983), 385-412. MR 85a:17004

[21] J. C. Jantzen, Moduln mit einem höchsten Gewicht, Springer, Berlin, Heidelberg, New York, 1979. MR 81m:17011

[22] A. Korányi and J. A. Wolf, Realization of hermitian symmetric spaces as generalized half planes, Ann. of. Math. 81 (1965), 265-288. MR 30:4980

[23] B. Krötz, Norm estimates for unitary highest weight modules, Ann. Inst. Fourier 49:4 (1999), 1242-1264. MR 2001i:22013

[24] B. Krötz, Formal dimension for semisimple symmetric spaces, Comp. Math. 125 (2001), 155-191. CMP 2001:09

[25] B. Krötz, K.-H. Neeb, and G. Ólafsson, Spherical representations and mixed symmetric spaces, Represent. Theory 1 (1997), 424-461. MR 99a:22031

[26] Neeb, K.-H., Holomorphy and Convexity in Lie Theory, de Gruyter, Berlin, 2000. MR 2001j:32020

[27] G. Ólafsson, Fourier and Poisson transformation associated to a semsisimple symmetric space, Invent. math. 90 (1987), 605-629. MR 89d:43011

[28] G. Ólafsson and B. Ørsted, The holomorphic discrete series for affine symmetric spaces. I, J. Funct. Anal. 81:1 (1988), 126-159. MR 89m:22021

[29] G. Ólafsson and B. Ørsted, The holomorphic discrete series of affine symmetric spaces and representations with reproducing kernels, Trans. Amer. Math. Soc. 326 (1991), 385-405. MR 91j:22014

[30] T. Oshima and T. Matsuki, A description of discrete series for semisimple symmetric spaces, Adv. Stud. Pure Math., vol. 4, North-Holland, Amsterdam, 1984, pp. 331-390. MR 87m:22042

[31] L. Schwartz, Théorie des distributions, Hermann, Paris, 1966. MR 35:730

[32] M. Vergne and H. Rossi, Analytic continuation of the holomorphic discrete series of a semisimple Lie group, Acta Math. 136 (1976), 1-59. MR 58:1032

[33] N. Wallach, The analytic continuation of the discrete series I, II, Tran. Amer. Math. Soc. 251 (1979), 1-17, 19-37. MR 81a:22009

Department of Mathematics, The Ohio State University, 231 West 18th Avenue, Columbus, Ohio 43210-1174

E-mail address: kroetz@math.ohio-state.edu

Fachbereich Mathematik, Technische Universität Darmstadt, Schlossgartenstr. 7, D-64289 Darmstadt, Germany

E-mail address: neeb@mathematik.tu-darmstadt.de 